\title{
Coordinated Control of Three-Phase AC and DC Type EV-ESS for Efficient Hybrid Microgrid Operations
}

\author{
Md Shamiur Rahman ${ }^{\mathrm{a}, 1, *}$, Md Jahangir Hossain ${ }^{\mathrm{a}, 2, * *}, J^{\prime}$ unwei $\mathrm{Lu}^{\mathrm{a}, 2}$ \\ ${ }^{a}$ Queensland Micro- and Nano Technology Centre, School of Engineering, Griffith University, Gold Coast, QLD-4222, \\ Australia
}

\begin{abstract}
The progressive deployment of electric vehicles (EV) and the increased penetration of alternating current $(\mathrm{AC})$ and direct current $(\mathrm{DC})$ type renewable resources paves the path towards hybrid AC/DC microgrid operation. Considering the potentials of EVs in the realm of the microgrids, this paper presents a three layered coordinated control strategy considering EV availability constraints for three-phase (3P) and DC type EV energy storage systems (EV-ESS) to improve the hybrid AC/DC microgrid operation. The first layer of the algorithm ensures DC subgrid management, which includes DC bus voltage regulation and DC power management. The second and third layer are responsible for the AC subgrid management, which include AC bus voltage and frequency regulation with active and reactive energy management. The multi-layered coordination is embedded into the microgrid central controller (MGCC) which controls the interlinking controller in between AC and DC microgrid as well as the interfacing controllers of the participating EVs and distributed renewable resources (DER). The hybrid AC/DC microgrid has been designed in MATLAB/SIMULINK ${ }^{\circledR}$ environment resembling the under construction microgrid at Griffith University, Australia. Extensive case studies have been performed considering real life irradiation data and commercial loads of the campus building. Impacts of homogeneous and heterogeneous single-phase EV charging are investigated to observe the unbalanced scenario. Synchronization state while switching from islanded to grid connected mode has also been tested considering contingency situation. From the comparative simulation results it is evident that the proposed controller exhibits effective and robust performance for all the cases.
\end{abstract}

Keywords: Hybrid AC/DC microgrid, Vehicle-to-grid (V2G), Electric vehicles (EVs), Energy storage, Photovoltaics (PV), Interlinking converter

\section{Introduction}

The accelerated depletion rate of the fossil fuels and the raising environmental concerns associated with the fossil-fired power generation are driving the global electricity generation systems towards distributed generators (DGs). Microgrids have the capability of clustering DGs such as renewable/sustainable resources and energy storage systems (ESS) through intelligent and coordinated control. As a result, the microgrid concept has been widely adopted which can provide several ancillary services such as grid voltage and frequency regulation, active and reactive power control, uninterruptable power supply (UPS) and fault ride through service during any grid fault. It can also contribute to enhance power quality and system reliability in an economic manner [1]. Based on the outputs of the participating DGs and loads, microgrids can be

\footnotetext{
${ }^{*}$ Corresponding author, Phone: +61474822325

** Principal corresponding author, Phone: +61432155461

Email addresses: mdshamiur.rahman@griffithuni.edu.au (Md Shamiur Rahman), j.hossain@griffith.edu.au

(Md Jahangir Hossain), j.lu@griffith.edu.au (Junwei Lu)

${ }^{1}$ Student Member, IEEE

${ }^{2}$ Senior Member, IEEE
} 
classified into AC, DC and hybrid AC/DC types. Each type of microgrid has its own characteristics, benefits and challenges associated with it. Currently, AC microgrid is predominant because of the AC nature of the existing grid. DC microgrid concept has recently been emerged in the distribution arena due to increased penetration of DC types DGs such as photovoltaic (PV), electric vehicles (EV) and so on. On the hand, hybrid $\mathrm{AC} / \mathrm{DC}$ microgrid has been proposed in some recent literatures [2]-[4] to combine the advantages of both AC and DC nature of the electric system. A hybrid AC/DC microgrid has several potential advantages over conventional AC and DC type microgrids, for instance the feasible integration of both AC and DC type DGs and loads with minimal AC/DC conversion, reduced requirement of synchronization due to the direct coupling of sources and loads to alike type common buses, simple voltage transformation using transformer for the $\mathrm{AC}$ side and DC/DC converters for DC sides, increased efficiency and reliability with improved economical operation due to less conversion loss [5]. Conceptual European super grid or so called mega-grid is the high level version of the hybrid $\mathrm{AC} / \mathrm{DC}$ microgrid which includes high voltage $\mathrm{AC}$ and $\mathrm{DC}$ transmission links to connect offshore wind turbines and desert based solar farms to serve the load consumption of the entire continent. Furthermore, commercial buildings consume approximately $32 \%$ of the worldwide energy usage. They hold accountable for about $30 \%$ of the total end-use energy-related $\mathrm{CO}_{2}$ emissions. As a result, commercial building can be operated with hybrid DGs such as PV and electric vehicle-energy storage systems (EV-ESS) by forming commercial hybrid AC/DC microgrids which can provide pivotal role in constructing green energy metropolitans $[6]-[8]$.

However, hybrid $\mathrm{AC} / \mathrm{DC}$ microgrid requires complex protection and control structures to ensure reliable operation, especially when the participating DGs are intermittent in behaviour like PV and wind turbines. Even though the advantages of predominant renewable sources like PV and wind turbines are widely acknowledged, their fluctuating outputs raises the problem of maintaining balance between electricity generation and demand. This issue can severely affect the system operation when the system operates in islanded mode. Tackling this issue with regulated AC and DC bus voltage and frequency requires the utilization of energy storages, such as battery energy storages (BESS) and EV-ESS.

The progressive deployment of electric vehicles in recent years has driven the Distribution System Operators (DSOs) to consider the implications and the advantages of using EV-ESS to low voltage (LV) networks. With proper utilization of EV-ESS, vehicle-to-grid (V2G) concept can improve the performance of the utility grids or microgrid in the area of efficiency, stability and reliability by offering ancillary services such as reactive power management, active power control, tracking the intermittent renewable energy resources, load balancing and shifting via valley filling support, peak load shaving and filtering current harmonics in the output. Although electric vehicle owners may obtain some benefit by individually providing ancillary services to the DSOs, V2G operation makes sense only when there is reasonably large number of EVs can take part into the operation. Aggregators or aggregation agents are intermediary authorities or computational entities that unify and coordinate distributed EV-ESS to provide medium to large-scale ancillary services [9]-[11]. However, due to various types of charging configurations such as DC and three-phase AC fast charging and single-phase AC slow charging make the aggregation of EVs a challenging task. Furthermore, increased penetration of single-phase or residential EV chargers introduce unbalanced phase voltage and current which may go beyond acceptable tolerance limit of the microgrid system.

Intensive ongoing research has been being performed in order to tackle the aforementioned issues. In [2] coordinated control of an islanded hybrid AC/DC microgrid with BESS, PV and wind turbine is presented. In [3] an autonomous control of the interlinking controller is presented by using normalized droop control. Both papers have emphasised on active power management even though during islanded condition reactive power management is equally necessary. Furthermore, the unbalanced condition has not been explored. Power management and control of a islanded and grid-connected hybrid AC/DC microgrid is presented in [12]. Robust power management with DC bus voltage regulation by exploiting ESS is explored in [13]. However, the grid synchronization has not been considered and more focus is given on the active power management. The feasibility of reactive power support through V2G operation is explored for single-phase and three-phase off-board chargers in [14], [15] respectively and the concept of Smartpark is introduced in [16], which has the capability of four quadrant V2G operation. However, a microgrid environment requires both active and reactive power sharing among multiple DGs. These requirements cannot be achieved by the aforementioned control strategies. A coordinated control is presented in [17] for frequency regulation only by 
Table 1: Salient features of the proposed approach in comparison with the contemporary literatures

\begin{tabular}{lcccccccccc}
\hline Considered issues & {$[\mathbf{2}]$,} & {$[\mathbf{1 2}]$,} & {$[\mathbf{1 6}]$} & {$[\mathbf{1 7}]$} & {$[\mathbf{1 8}]$} & {$[\mathbf{1 9}]$} & {$[\mathbf{2 0}]$} & {$[\mathbf{2 1}]$} & $\begin{array}{c}\text { This } \\
\text { paper }\end{array}$ \\
\hline Concurrent AC/DC voltage regulation & $\checkmark$ & $\checkmark$ & & & & & & & $\checkmark$ \\
Frequency regulation & $\checkmark$ & $\checkmark$ & & $\checkmark$ & $\checkmark$ & $\checkmark$ & & $\checkmark$ & $\checkmark$ \\
Single-phase EV charging & & & & & $\checkmark$ & $\checkmark$ & $\checkmark$ & & $\checkmark$ \\
$\begin{array}{l}\text { Four quadrant V2G operaiton } \\
\text { Grid synchronization }\end{array}$ & & & $\checkmark$ & & & $\checkmark$ & & $\checkmark$ & $\checkmark$ \\
\hline
\end{tabular}

incorporating large scale EVs through monitoring and controlling of the area control signal (ACS). The role of EVs in microgrid service restoration by black starting is explored in [18]. This paper has also explored the active power sharing capability using conventional droop control. However, the conventional droop control mechanism is not able to provide enough damping to stabilize the voltage and frequency. Stability analysis has been performed in [19] for asymmetrical virtual inertia caused by the single-phase EV charges in a droop based weak grid or microgrid. The paper has also shown that the conventional droop control is unable to provide accurate frequency regulation. Tackling the unbalanced distribution grid using coupled PV inverter and EV chargers is presented in [20]. The primary focus is given to explore the potential of EVs of balancing phase voltages which in turns reduce the unbalanced condition.

Motivated by the ongoing research and the aforementioned scopes of improvements, this paper presents a unified approach which include the design of an interlinking/interfacing controller with four quadrant operational capability and a three layered coordinated control strategy for three-phase and DC type EV-ESS taking EV availability constraints into account to improve the performance of an islanded commercial hybrid AC/DC microgrid. The first layer of the algorithm manages the DC subgrid. The main responsibility of this layer is to ensure DC bus voltage regulation and the power management within the DC subgrid. The second and third layers are accountable for the management of the AC subgrid. Both layers simultaneously operate to ensure AC bus voltage and frequency regulation within the AC subgrid. The designed coordination algorithm is embedded into the microgrid central controller (MGCC) as a single entity. The MGCC is responsible to generate necessary power management commands for the local controllers (LC). LCs such as interlinking/interfacing controllers receive these commands and perform required actions on smart inverters. The hybrid AC/DC microgrid and the controllers are designed and tested in MATLAB/SIMULINK ${ }^{\circledR}$ environment. The hybrid AC/DC microgrid is designed considering the under construction microgrid structure at Griffith University, Australia. In order to validate the robust performance of the coordinated control various case studies have been performed. These case studies include the performance and the impact analysis of the designed controllers under heterogeneous and homogeneous charging of single-phase EVs with variable commercial loads and solar irradiation. Furthermore, considering the contingency condition, the controller has been tested from islanded to grid connected mode as well. Table 1 shows the comparison of the proposed approach with the contemporary literatures regarding microgrids and V2G. The main contributions of this paper can be summarized as follows,

- Concurrent variable PV output due to intermittent irradiation and variable real commercial load profile has been considered.

- A four-quadrant alike structured interlinking and interfacing LCs has been designed. The designed LCs can regulate both active and reactive power by exploiting the four-quadrant operational capability of the V2G operation.

- A multi-layered coordination algorithm has been proposed for the MGCC in order to coordinate threephase (3P) and DC type EV-ESS considering their availability constraints. The layered coordination ensures optimum energy management by prioritizing autonomous DC and AC microgrid operation without unnecessary power flow caused by the hybrid operation 


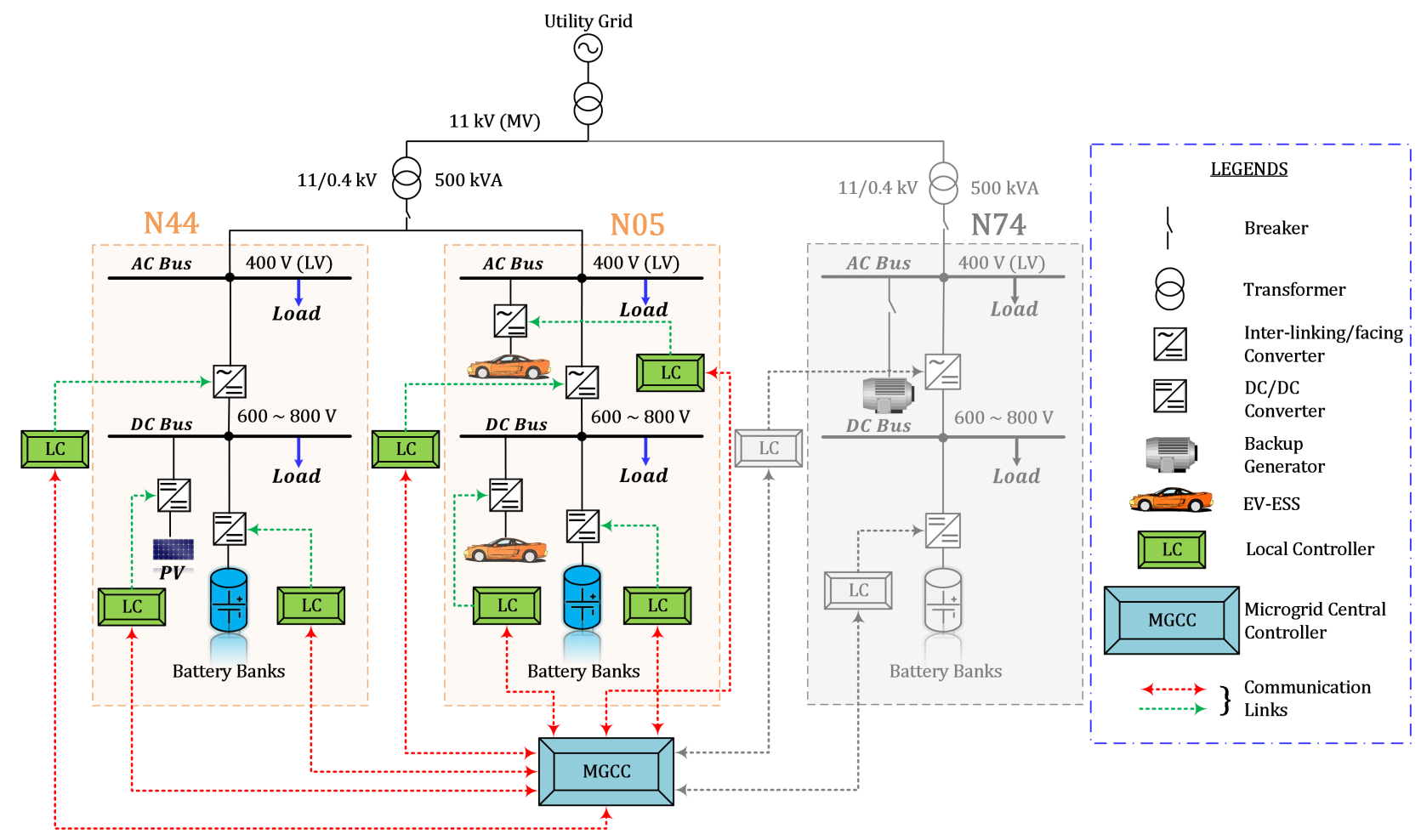

Figure 1: Hybrid AC/DC microgrid structure in Griffith University, Australia.

- Simultaneous AC and DC bus voltage and system frequency regulation has been ensured in order to obtain improved hybrid AC/DC operation. Unlike the previous literatures [2], [3], [13] unbalanced condition has also been considered caused by single-phase EV chargers.

The rest of the paper is organized as follows. Section 2 gives a brief introduction of the under construction hybrid AC/DC microgrid in Griffith University, Australia. Later, dynamic model of the PV, EV-ESS, the developed interlinking/interfacing controller and DC/DC converter controller are presented sequentially under the umbrella of Section 2. Coordinated control structure with its objectives and step-by-step algorithm is presented in Section 3. The proposed control scheme is verified by simulation. The case studies of the simulation results are presented and elaborately discussed in Section 4. Section 5 draws the conclusion for the paper. Appendix A presents the simulation parameters for the designed hybrid AC/DC microgrid and its controllers. Appendix B shows illustrations with description tables for the testing facility equipments.

\section{Hybrid AC/DC microgrid}

The test hybrid AC/DC microgrid system has been designed based on the under developed microgrid at the Griffith University, Australia. The microgrid includes three buildings, namely N44, N05 and N74 as shown in Fig. 1 [22]. Each building operates as an individual smart hybrid AC/DC microgrid with separate $\mathrm{AC}$ and DC buses. Each AC and DC bus of these buildings accommodate separate AC and DC sources and loads. Every pair of AC and DC buses are interfaced with the bidirectional interlinking distribution static synchronous compensators (D-STATCOM) or smart voltage source inverters (VSI) [23].

N44 building contains PV panels of $16 \mathrm{KWp}$ with $60 \mathrm{kWh}$ Lithium-ion polymer (Li-ion) BESS [24]. The BESS are connected to the corresponding DC bus through an intelligent DC/DC converter. The benefits of having BESS attached to the PV system are twofold. Primarily, the BESS will tightly regulate the output voltage of the PV system and secondarily, it will buffer the intermittent PV output power [22]. The combined 
DG unit (PV and BESS) is presently used for the peak demand management purpose. The capacity of these DGs will be increased in the future based on the total load demand to utilize them in microgrid operation within one or multiple buildings.

N05 building is allocated for smart EV/plug-in hybrid electric vehicle (PHEV) charging station, which will facilitate V2G operation. EV-ESSs will be charged using bidirectional DC fast chargers when they are connected to the DC bus. AC charging is planned to be installed at the AC bus of the N05 building. The bidirectional AC charging will include on-board three-phase fast charging and single-phase slow charging. N74 building contains sensitive loads attached to both buses. This building requires an uninterruptible power supply (UPS) operation due to the presence of critical loads like laboratory equipment and testing samples. The building is presently served by backup diesel generator and battery energy storages during outage. The diesel generators are inherently slower compared to the micro-turbines due to the high inertia. As a result, the backup diesel generators will be replaced in the future with micro-turbines for their fast starting capability.

In order to monitor and analyse data sensor-based smart power meter (Power Meter PM5350A) has been installed in each of the buildings. Sensor-based data have been collected using OSI PI software using a centralized communication structure in order to monitor and control the microgrid operation. Modbus TCP/IP (Transmission Control Protocol/Internet Protocol) has been used as the communication protocol for supervisory control and data acquisition (SCADA) systems. Hypertext Transfer Protocol (HTTP) TCP/IP communication protocol has been used as the backup protocol when the Modbus TCP/IP is not available [25]. Microgrid central controller (MGCC) is responsible for generating command signals for the LCs. These command signals are transmitted through Modbus TCP/IP communication protocol to the remote terminal units (RTU) such as LCs of D-STATCOMs, DC/DC converters etc.

In this paper the primary concern is directed towards the microgrid operation of N44 and N05. As a result, the simulation model has been designed comprising only N44 and N05 microgrid system. The model of N74 is not included as shown in the shaded portion in Fig. 1. Both islanded and grid connected operation of the buildings have been taken into consideration. The capacity of the DG units has been adjusted comparing with the real load profile of the buildings. For the simulation purpose $250 \mathrm{~kW}$ PV with $60 \mathrm{kWh}$ high capacity energy storage systems are used. PV module comprises of PV panels and maximum power point tracking (MPPT) system integrated boost converter. Perturb and observe ( $\mathrm{P}$ and $\mathrm{O}$ ) algorithm has been chosen for the MPPT system. BESS are attached nearby through separate DC/DC converter which is controlled by charge/discharge algorithm. Intelligent DC fast charging has been designed in N05 building with EV-ESS and DC/DC converter and connected to the DC bus. Single-phase AC and three-phase AC charging has been modelled with EV-ESS and associated bidirectional single-phase and three-phase inverters respectively. Both DC buses of N44 and N05 buildings are interlinked with corresponding AC buses through interlinking VSIs and LCL filters. A concise modelling of the DG units and the control strategies used in the design are presented in consequent subsections. Necessary parameters related to the model and controller are provided in Appendix A.

\subsection{PV module modelling}

Using the physical property of the $p-n$ semiconductor, the model of PV module can be obtained based on the PV array current-voltage relationship presented in (1)

$$
i_{p v i}=N_{p i} I_{L i}-N_{p i} I_{s i}\left[\exp \left\{\alpha_{p i}\left(\frac{V_{p v i}}{N_{s i}}+\frac{R_{s i} i_{p v i}}{N_{p i}}\right)\right\}-1\right]-\frac{N_{p i}}{R_{s h i}}\left(\frac{V_{p v i}}{N_{s i}}+\frac{R_{s i} i_{p v i}}{N_{p i}}\right)
$$

Where, $I_{L i}=$ current corresponding to light

$I_{s i}=$ reverse saturation current chosen as $9 \times 10^{-11} \mathrm{~A}$

$N_{s i}=$ the number of cells in series

$N_{p i}=$ the number of modules in parallel

$R_{s i}$ and $R_{s h i}=$ series and shunt resistances of the array

$i_{p v i}=$ the current flowing through the array 
$V_{p v i}=$ the output voltage of the array

The constant $\alpha_{p i}=\frac{q_{i}}{A_{i} k_{i} T_{r i}}$

Where, $k_{i}=1.3807 \times 10^{-23} \mathrm{JK}^{-1}$ is the Boltzmann constant

$q_{i}=1.6022 \times 10^{-19} \mathrm{C}$ is the charge of an electron

$A_{i}=$ the $p-n$ junction ideality factor with a value between 1 and 5 ,

and $T_{r i}=$ the cell reference temperature

To extract the maximum power from the PV module different MPPT systems, for instance perturb and observe (P \& O), incremental conductance (IC), hill climbing etc. are usually used. For this paper perturb and observe method has been chosen due to ease in implementation and reasonably satisfactory performance $[26]-[28]$.

\subsection{EV Energy storage modelling}

Li-ion based battery energy storage has been used to design both battery banks and EV-ESS battery packs in the hybrid AC/DC microgrid. Generic third order Thevenin equivalent circuit model presented in [29] has been used to model the battery. The only difference in designing the battery banks and the EVESS battery pack is that the EV-ESS battery pack possess the higher $\mathrm{kWh}$ capacity than ordinary battery banks. Both types of energy storage systems are interfaced with DC/DC converter. The DC/DC converter executes the charge and discharge command generated by LC or MGCC. Both battery banks and DC type EV-ESS are directly connected to the DC bus through individual DC/DC converters. For three-phase or single-phase AC charging the EV-ESS battery packs coupled with associated DC/DC converters are later connected to the three-phase or single-phase inverters respectively. The dynamic charging and discharging of Li-Ion battery model is designed based on (2) and (3) respectively [30], [31].

For $i^{*}>0$ the battery will act as in discharge mode which can be represented as

$$
f_{1}\left(i t, i^{*}, i\right)=E_{0}-K \frac{Q}{Q-i t} i^{*}-K \frac{Q}{Q-i t} i t+A e^{-B \times i t}
$$

For $i^{*}<0$ the battery will act as in charge mode which can be represented as

$$
f_{1}\left(i t, i^{*}, i\right)=E_{0}-K \frac{Q}{i t+0.1 Q} i^{*}-K \frac{Q}{Q-i t} i t+A \cdot e^{-B \times i t}
$$

Where, $E_{0}=$ Constant voltage $(V)$

$K=$ Polarization constant $(A h)^{-1}$ or Polarization resistance $(\Omega)$

$i^{*}=$ Low frequency current dynamics $(A)$

$i=$ Battery current $(A)$

$i t=$ Extracted capacity $(A h)$

$Q=$ Maximum battery capacity $(A h)$

$A=$ Exponential voltage $(V)$

$B=$ Exponential capacity $(A h)^{-1}$

\subsection{Interlinking/interfacing converter control}

From the controlling point of view, the most important part of a hybrid AC/DC microgrid is the interlinking converter controller which links the DC bus with the AC bus. The interlinking converter controller consists of three inner loop controllers for both islanded and grid connected operation of the microgrid. These inner control loops are power controller, voltage controller and current controller. The dynamic modelling for each of the control loops are derived and presented in the succeeding subsections. 


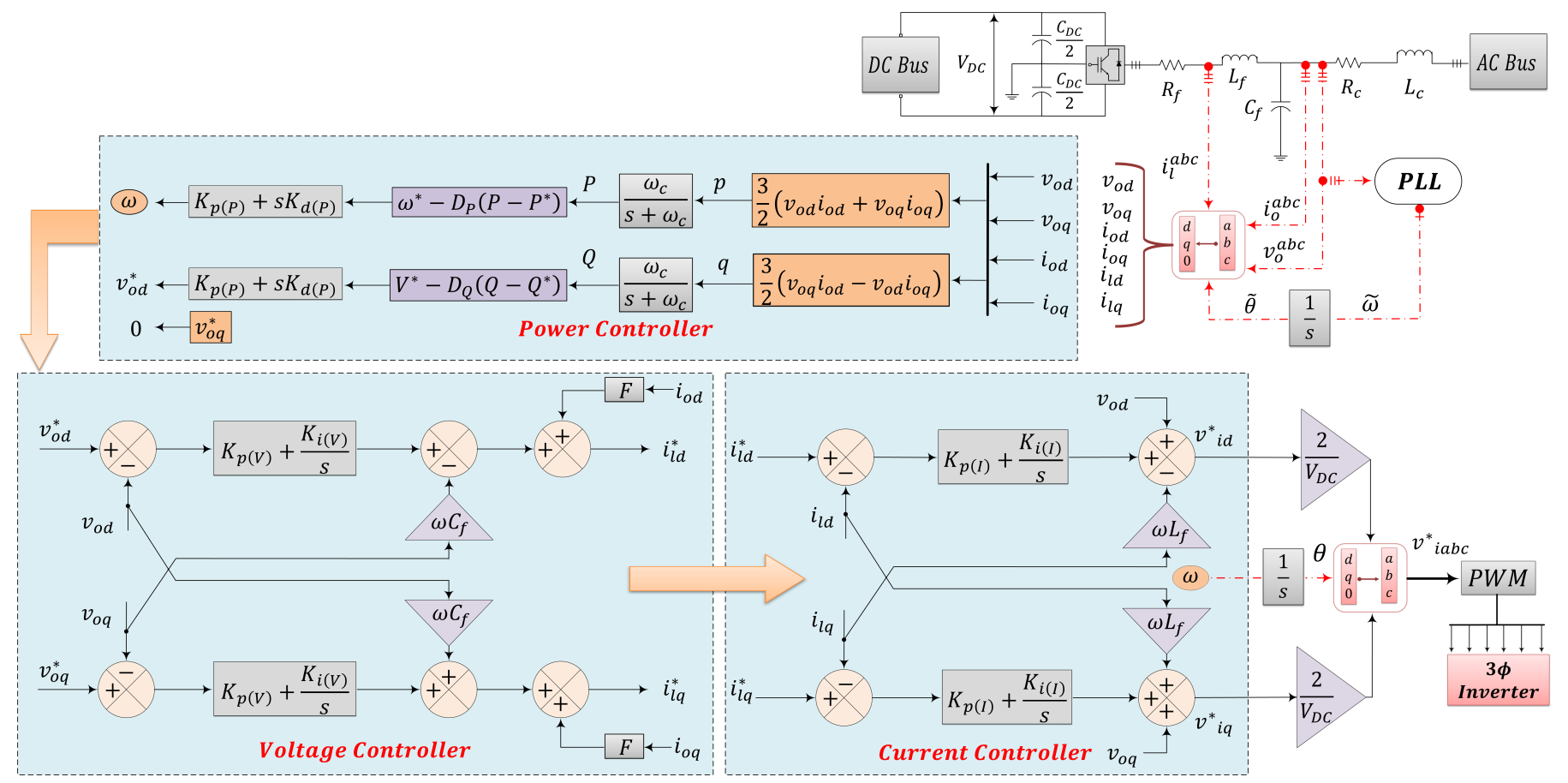

Figure 2: Modified local control structure for each interlinking/interfacing converters of the hybrid AC/DC microgrid

\subsubsection{Power Controller}

The power controller is shown in Fig. 2. Instantaneous voltage $\left(v_{o}^{a b c}\right)$ and current $\left(i_{o}^{a b c}\right)$ after the LC filter and the inverter output current $\left(i_{l}^{a b c}\right)$ are measured and transformed into their corresponding $d-q$ components (denoted by separate subscripts in $d$ and $q$ ). The real and reactive power can be calculated from the measured line voltage and current components by using (4) [32].

$$
\left\{\begin{array}{l}
p=\frac{3}{2}\left(v_{o d} i_{o d}+v_{o q} i_{o q}\right) \\
q=\frac{3}{2}\left(v_{o q} i_{o d}-v_{o d} i_{o q}\right)
\end{array}\right.
$$

Fundamental components of the calculated real and reactive power are extracted using a low pass filter $(\mathrm{LPF})$ with cutoff frequency $\omega_{c}$. The outputs of the LPF are the inputs for the droop control as shown in (5)

$$
\left\{\begin{array}{l}
P=\frac{\omega_{c}}{s+\omega_{c}} p \\
Q=\frac{\omega_{c}}{s+\omega_{c}} q
\end{array}\right.
$$

Voltage and frequency regulation during islanded microgrid operation requires specific control actions. These requirements can be achieved through an embedded droop control mechanism within VSI LCs. The main concept of the droop mechanism is to mimic the characteristics of synchronous generators which include reduced angular velocity with increased load demand. Generally the $X / R$ ratio of a microgrid is very high due to the high value of the inductors present in LC filters. As a result of this inductive nature of a microgrid, well known $P / \omega$ and $Q / V$ droop control can be applied to regulate the frequency and the voltage. The conventional droop control scheme is shown in (6) [33]. 


$$
\left\{\begin{array}{l}
\omega=\omega^{*}-D_{P}\left(P-P^{*}\right) \\
V=V^{*}-D_{Q}\left(Q-Q^{*}\right)
\end{array}\right.
$$

Where, $D_{P}$ and $D_{Q}$ are the droop coefficients for $P / \omega$ and $Q / V$ droop controller respectively. "*" represents the reference signals of the corresponding variables. The $d-q$ reference frame is rotating synchronously with the frequency of the line voltage measured by the phase-locked-loop (PLL). As a result, $v_{o q}^{*}$ is taken 0 . The conventional droop mechanism possesses inherent trade-off between active and reactive power sharing accuracy and frequency and amplitude regulation. Considering this issue a modified proportional-derivative (PD) compensated droop control has been utilized according to [34]. The PD compensated droop control ensures proper damping and desirable power sharing accuracy with adequate frequency and voltage regulation. The droop coefficients can be calculated using the following equations,

$$
\left\{\begin{array}{l}
D_{P}=\frac{2 \pi\left(f_{\max }-f_{\min }\right)}{P_{\max }} \\
D_{Q}=\frac{V_{\max }-V_{\min }}{Q_{\max }}
\end{array}\right.
$$

Considering that the three-phase inverter interfaced EV chargers are V2G capable and can absorb or supply both real and reactive power. Then the above droop coefficients for the three-phase inverter interfaced EV-ESS would be [35],

$$
\left\{\begin{array}{l}
D_{P}=\frac{2 \pi\left(f_{\max }-f_{\min }\right)}{2 P_{\max }} \\
D_{Q}=\frac{V_{\max }-V_{\min }}{2 Q_{\max }}
\end{array}\right.
$$

According to the Australian Energy Market Operator (AEMO) specification, the acceptable frequency range for islanded microgrid operation in Australia is in between $49.5 \mathrm{~Hz}$ and $50.5 \mathrm{~Hz}$ [36], [37] and the voltage magnitude limit lies within $-6 \%$ to $+10 \%$ [38]. So the $f_{\max }, f_{\min }, V_{\max }$ and $V_{\min }$ have been taken as specified standard. By observing the load profile maximum KVA ratings for both interlinking converters have been chosen.

\subsubsection{Voltage controller}

The differential algebraic equations (DAE) for the voltage controller have been derived as shown in (9) and (10) [35], [39]-[41]

$$
\left\{\begin{array}{l}
\dot{\phi_{o d}}=v_{o d}^{*}-v_{o d} \\
\dot{\phi_{o q}}=v_{o q}^{*}-v_{o q}
\end{array}\right.
$$

$$
\left\{\begin{array}{l}
i_{l d}^{*}=F i_{o d}-\omega C_{f} v_{o q}+K_{p(V)} \dot{\phi_{o d}}+K_{i(V)} \phi_{o d} \\
i_{l q}^{*}=F i_{o q}-\omega C_{f} v_{o d}+K_{p(V)} \dot{\phi_{o q}}+K_{i(V)} \phi_{o q}
\end{array}\right.
$$

Where $\dot{\phi_{o d}}$ and $\dot{\phi_{o q}}$ are state variables corresponding to the proportional-integral (PI) controller of the voltage controller. "*" represents reference signal of corresponding $d$ and $q$ components of voltage and current. $\omega$ is the angular frequency generated by the droop control. $K_{p(V)}$ and $K_{i(V)}$ are respectively proportional and integral gain. $F$ is the feed-forward gain also known as the virtual impedance. $F$ can be calculated from the line and filter resistances.

\subsubsection{Current controller}

The DAEs for the voltage controller are presented in (11) and (12) [35], [39]-[41]

$$
\left\{\begin{array}{l}
\dot{\lambda_{l d}}=i_{l d}^{*}-i_{l d} \\
\dot{\lambda_{l q}}=i_{l q}^{*}-i_{l q}
\end{array}\right.
$$




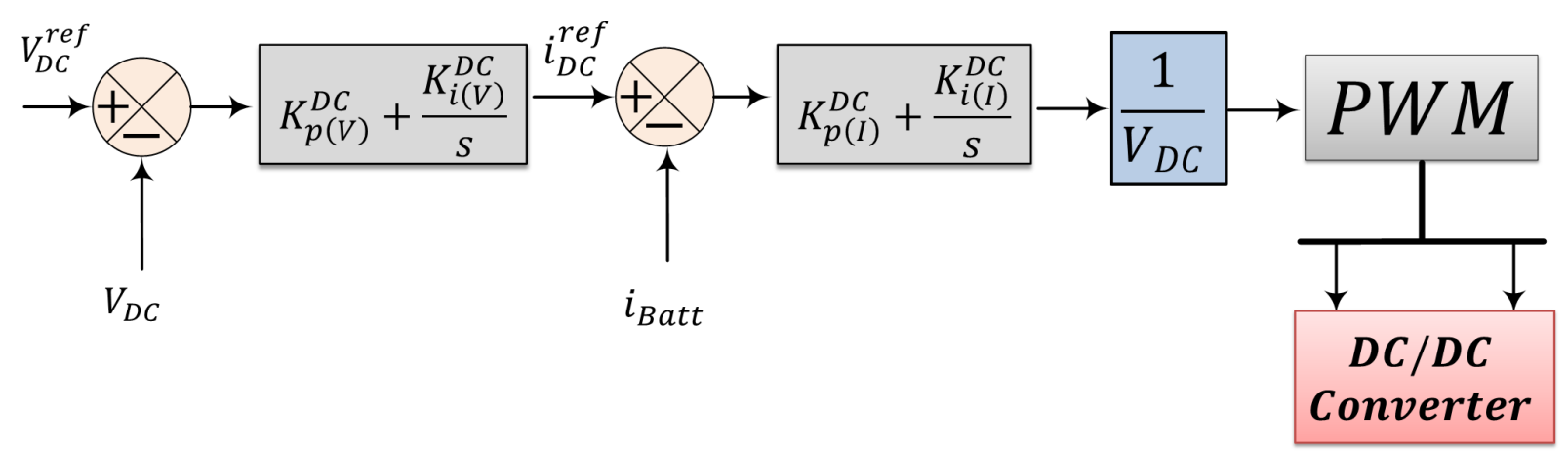

Figure 3: DC/DC converter controller

$$
\left\{\begin{array}{l}
v_{i d}^{*}=v_{o d}-\omega L_{f} i_{l q}+K_{p(I)} \dot{\lambda_{l d}}+K_{i(I)} \lambda_{l d} \\
v_{l q}^{*}=v_{o q}+\omega L_{f} i_{l d}+K_{p(I)} \dot{\lambda_{l q}}+K_{i(I)} \lambda_{l q}
\end{array}\right.
$$

Where $\dot{\lambda_{l d}}$ and $\dot{\lambda_{l q}}$ are state variables corresponding to the proportional-integral (PI) controller of the current controller. "*" represents reference signal of corresponding $d$ and $q$ components of voltage and current. $K_{p(I)}$ and $K_{i(I)}$ are respectively proportional and integral gain. The conventional current controller has been modified by feed-forwarding the line voltage components to get improved performance.

\section{4. $D C / D C$ converter control}

The DC/DC converter is the interfacing converter for battery banks and EV-ESS battery packs as illustrated in Fig. 3. The DC/DC converter includes a voltage control loop cascaded with a current control loop. The voltage control loop keeps the DC link voltage of the energy storage between VSI or DC bus at $V_{D C}^{r e f}$ and generate reference current signals for the battery. DAEs for the voltage and current control loop of the DC/DC converter controller are shown in (13) [42]

$$
\left\{\begin{aligned}
i_{D C}^{r e f} & =\left(V_{D C}^{r e f}-V_{D C}\right)\left(K_{p(V)}^{D C}+\frac{K_{i(V)}^{D C}}{s}\right) \\
D_{B a t t} & =\frac{\left(i_{D C}^{r e f}-i_{B a t t}\right)\left(K_{p(I)}^{D C}+\frac{K_{i(I)}^{D C}}{s}\right)}{V_{D C}}
\end{aligned}\right.
$$

Where $K_{p(V)}^{D C}$ and $K_{i(V)}^{D C}$ are the proportional and integral gains for the voltage control loop respectively and $K_{p(I)}^{D C}$ and $K_{i(I)}^{D C}$ are the proportional and integral gains for the current control loop respectively. $D_{B a t t}$ is the generated duty ratio for the pulse width modulation (PWM) of the DC/DC converter.

\section{Coordinated control strategy}

\subsection{Coordinated control objectives}

The coordinated control of the three-phase and the DC type EV-ESS is designed considering following objectives:

- Bus voltage regulation: Hybrid AC/DC microgrids are composed of single or multiple AC and DC buses. It is essential to maintain both $\mathrm{AC}$ and $\mathrm{DC}$ bus voltage levels within an acceptable range to maintain stable hybrid AC/DC microgrid operation. Usually, during islanded condition the amplitude and frequency of the AC bus voltage are regulated by the bidirectional interlinking converter and the 
DC bus voltage is controlled by the DC/DC converter connected to energy storage devices. According to [43] the input DC bus voltage and the output AC bus RMS voltage of the interconnecting VSI maintains a linear relationship as shown in (14)

$$
V_{D C}=2 \times \underbrace{\left(\frac{\Lambda}{m}\right) \times\left(\frac{\sqrt{2}}{\sqrt{3}}\right)} \times V_{L-L}
$$

Where, $V_{D C}=\mathrm{DC}$ bus voltage;

$V_{L-L}=$ Peak-to-peak magnitude of the phase-to-phase voltage;

$\Lambda=$ voltage compensating factor for the VSI interconnection loss;

$m=$ modulation index;

Considering the loss less interconnection of the VSI (i.e. $\Lambda=1)$ and the maximum value of the modulation index (i.e.m $=1)$ then the $(14)$ can be rewritten as follows

$$
V_{D C} \approx 2 \times \underbrace{\left(\frac{\sqrt{2}}{\sqrt{3}}\right) \times V_{L-L}}_{V_{L-N}^{R M S}}
$$

It can be observed from (15) that the DC bus voltage needs to be double in magnitude of the phaseto-neutral RMS voltage (i.e. $V_{L-N}^{R M S}$ ). As mentioned previously that based on AEMO standard the AC bus RMS voltage should be in between $-6 \%$ to $+10 \%$. Due to the linear relation between DC bus voltage and AC bus RMS voltage, the acceptable deviation for DC bus voltage should be within $-3 \%$ to $+5 \%$. This defined relation has been utilized for the medium voltage (MV) DC microgrid in [44], however, this relationship must be applied to the low voltage (LV) distribution networks as well.

- Frequency regulation: During grid connected operation the frequency of the AC subgrid is regulated by the utility grid itself. However, islanded operation requires The frequency of the output voltage of each VSI or interlinking converter to be adjusted through the embedded droop control of the associated VSIs.

- Power sharing: During grid-tied mode, DG sources generate pre-specified active/reactive power and the main utility grid act as slack bus. Throughout this operation, the main objective of the utility grid is to provide or absorb power to balance the frequency by managing loads. The challenging condition arises when microgrids operate in islanded operation. During islanded operation microgrids are solely responsible for managing the total load demand by proportional sharing of real and reactive power.

- Reduction of phase unbalance: The introduction of single-phase or residential charger for the EVs will generate unbalanced phase voltage and current. With increased penetration of EVs this unbalanced condition might go beyond acceptable range, which require optimal coordination among EV-ESS aggregators of various configurations. This paper considers the generation of separate sinusoidal modulation indexes for each phases to partially tackle this issue. However, proper load planning from the DSOs is required to distribute the single-phase loads among phases in the case of the higher unbalanced condition.

\subsection{Coordinated control algorithm}

During normal operation of the microgrid it is expected that the overall demand of active and reactive power is balanced with the respective generation. Any mismatch in total generation and load demand requires regulation services. Usually DC bus voltage magnitude and the frequency of the AC bus voltage is closely related to the balance of active power generation and load demand. If the active power generation is higher than the load demand, then the excess active power will increase the value of the frequency and the DC bus voltage. As a result a regulation down service is required for both DC bus voltage and frequency 
of the system. Regulation down service involves routing the energy to the grid or charge the energy storage system which are not taking part in the microgrid operation. Regulation-up service are required when there is a shortage of generating active power than load. Then additional active power demand will decrease the DC bus voltage and at the same time the frequency of the system. Regulation-up service involves importing additional power from the grid (grid connected mode) or from the participating ESS (in islanded condition) which results in the discharging mode of the ESS.

On the contrary, for a converter dominated microgrid with relatively high value of the inductors in the LC or LCL filters, the magnitude of the AC bus RMS voltage is regulated by matching the reactive power generation and demand. Similar to the DC bus voltage and frequency regulation, if the reactive power generation is higher than the total reactive load, it will increase the magnitude of the AC bus RMS voltage, as a result regulation-down service is required. On the other hand, if the reactive power generation is less than the total reactive load, then the magnitude of the AC bus RMS voltage will decrease which requires regulation-up service. Regulation-up or down of the AC bus voltage magnitude can be obtained by absorbing or supplying the reactive power from or to the grid or from/to the four quadrant STATCOM attached to DGs or distributed energy storages like EV-ESS.

Synchronous generators/alternators run by natural gas and coal units can provide regulation-up or regulation-down for all essential parameters, by reducing their production or by supplying additional power to the grid, respectively, to match the load. Due to their slower dynamics and environmental impacts the fast responding converter based DGs and ESS are preferred in this paper. Due to comparative higher expenses of ESS they require smart coordination to be utilized. As a result, this paper proposes a smart coordination algorithm that can effectively provide satisfactory performance for the hybrid AC/DC microgrid for both grid connected and islanded operation even in the presence of single-phase EV-ESS charging station.

The proposed coordination algorithm is illustrated in Fig. 4. The smart coordination algorithm has considered the operation of the discharging mode of the DC fast charging station and the four quadrant operation of the three-phase charging station in the presence of single-phase AC charging for the electric vehicles. The coordination algorithm has three layers. It should be noted that all three layers can operate concurrently and autonomously without any overlapping factors in order to improve hybrid AC/DC microgrid operation. The advantages of simultaneously operating multi-layered coordination are, for instances, it will avoid excessive power transport due to hybrid operation, which results in lower power electronic based loss and it will also ensure the highest possible autonomy to the individual AC and DC microgrid. The notations presented in the Fig. 4 have following meanings,

- $P_{D C}^{M G} \rightarrow P_{A C}^{M G}$ : Active power is flowing through the interlinking converter from the DC microgrid to the $\mathrm{AC}$ microgrid

$-P_{D C}^{M G} \leftarrow P_{A C}^{M G}:$ Active power is flowing through the interlinking converter from the AC microgrid to the DC microgrid

$-Q_{D C}^{M G} \rightarrow Q_{A C}^{M G}$ : Reactive power is flowing through the interlinking converter from the DC microgrid to the $\mathrm{AC}$ microgrid

$-Q_{D C}^{M G} \leftarrow Q_{A C}^{M G}$ : Reactive power is flowing through the interlinking converter from the AC microgrid to the $\mathrm{DC}$ microgrid

$\Delta V_{D C}$ and $\Delta V_{A C}$ denote the deviation of the $\mathrm{DC}$ and $\mathrm{AC}$ bus voltages from their corresponding nominal values.

Layer 01: The first layer is responsible for the DC subgrid management and the DC bus voltage regulation. This layer prioritized the DC side load management by coordinating the DC EV-ESS. Initially this control layer will check whether the DC bus voltage requires regulation-up or regulation-down service by instantaneously checking the DC bus voltage magnitude and comparing that value with the allowable range. If the $\mathrm{DC}$ bus voltage is within acceptable range, then it will check whether AC subgrid requires additional active power. If the required power by the AC subgrid is within the acceptable KVA rating of the interlinking converter then it will export power to the AC subgrid. On the other hand, if the DC bus voltage requires regulation down service, then it will charge the DC EV-ESS battery based on the requirement or no control action will be taken as shown in the illustration. For the regulation-up service this control initiates the EV-ESS discharging operation. The layer will prioritize the initiation of DC EV-ESS over 3P EV-ESS based on the availability. Later, if necessary it can import/export additional power to/from the AC 


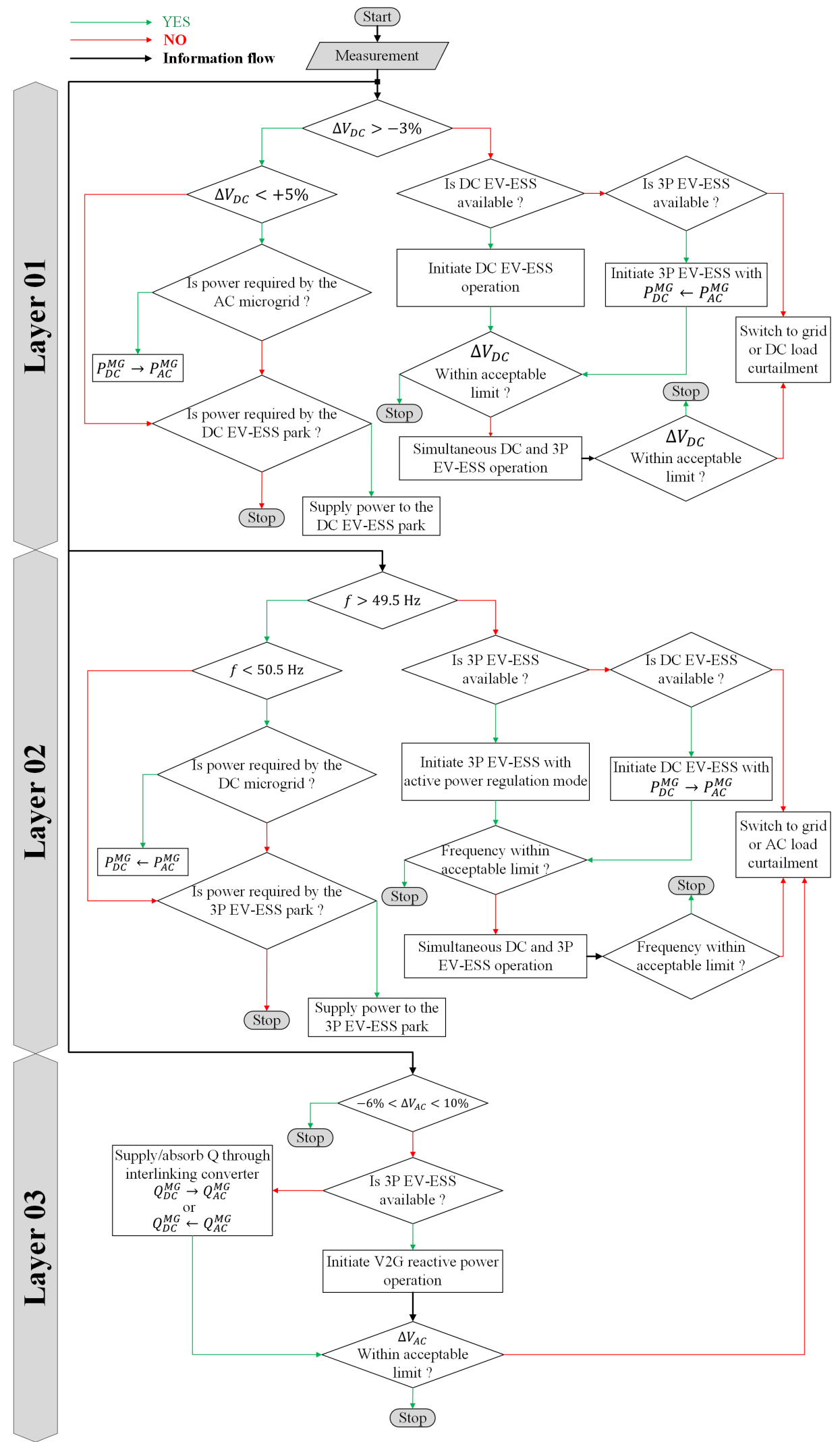

Figure 4: Proposed three layered coordination algorithm 
subgrid through the interlinking converter by initiating 3P EV-ESS operation based on their availability. For contingency purpose if even with the simultaneous DC and 3P EV-ESS operation the DC bus voltage cannot be regulated within the limit, DC load curtailment for the islanded condition or switching back to the grid has been suggested.

Layer 02: The second and third layer are dedicated to AC subgrid management. The second layer is closely resembled to the first layer as both parameters i.e. DC bus voltage and the frequency are closely related to the active power regulation. The exemption between Layer 01 and Layer 02 is the prioritized choice of operating 3P EV-ESS over DC EV-ESS due to their close proximity and configuration in the AC subgrid.

Layer 03: The third layer manages the reactive power of the AC subgrid to regulate the AC bus RMS voltage. This layer changes the reactive power references of the control system of the interlinking converters or the 3P EV-ESS interfacing converter. This layer will initially look for reactive power support (either source or sink of reactive power) from the 3P EV-ESS. However, if no reactive power support can be obtained from the 3P EV-ESS then it will send the required reactive power signal to the interlinking converter droop controller to supply or absorb additional reactive power.

It is not expected that EVs are allowed to be available all the time compared to any other static ESS such as BESS. Moreover, economical aspects of the application of large scale EV-ESS in distribution level is still a major research topic. Apart from these issues, optimum utilization of V2G operation requires a rigorous market framework. As the primary focus of this paper is inclined to the design of the coordination and control strategy of various types of EV-ESS, the details about the possible market framework for V2G operation are not discussed here. However, EV availability constraints which are relevant to the design process of the proposed controller are presented in the next section.

\subsubsection{EV availability constraints:}

- EV-ESS capacity constraints: Overcharging or discharging has adverse effect on EV battery lifetime. As a result, charging/discharging of both DC or 3P EV-ESS need to satisfy energy constraints mentioned below:

$$
\left(S O C_{i}^{\text {max }}-S O C_{i}^{0}\right) Q_{i}^{\text {Rated }} \leq \Delta Q_{i}^{\Delta T_{i}} \leq\left(S O C_{i}^{0}-S O C_{i}^{\text {min }}\right) Q_{i}^{\text {Rated }}
$$

Where,

$$
\left\{\begin{aligned}
\Delta Q_{i}^{\Delta T_{i}} & =\Delta T_{i} P_{i} \\
\Delta T_{i} & =T_{i(\text { final })}-T_{i(\text { initial })}
\end{aligned}\right.
$$

In the above equations,

$S O C_{i}^{\max }$ and $S O C_{i}^{\min }$ are the maximum and minimum allowable SOC for the $i$ th EV;

$S O C_{i}^{0}$ is the initial SOC of the $i$ th EV before participation;

$Q_{i}^{\text {Rated }}$ is the rated battery capacity in ampere-hour (Ah) of the $i$ th $\mathrm{EV}$;

$\Delta T_{i}$ is the charging/discharging duration of the $i$ th $\mathrm{EV}$;

$\Delta Q_{i}^{\Delta T_{i}}$ is the change in battery capacity within $\Delta T_{i}$ duration of the $i$ th $\mathrm{EV}$

- EV user requirements: In order to ensure flexible user experiences, the EV user should be able to pre-set the available time and minimum required SOC value for their EVs:

$$
\begin{aligned}
& T_{i} \epsilon\left\{T_{i(\text { initial })}, T_{i(\text { final })}\right\} \\
& S O C_{i}^{\text {final }} \geq S O C_{i}^{\text {pre-set }}
\end{aligned}
$$

Where,

$T_{i}$ is the available or controllable time of $i$ th $\mathrm{EV}$, during the period that EV can be involved in V2G service;

$T_{i(\text { initial })}$ and $T_{i(\text { initial })}$ are the allowable start and stop time (charging or discharging) of $i$ th EV 
respectively. The start and stop time of charging and discharging are dependent on several factors such as time of use pricing, driving patterns of EV owners etc. $S O C_{i}^{\text {final }}$ and $S O C_{i}^{\text {pre-set }}$ are the battery SOC value after its participation for V2G service the minimum SOC required value of $i$ th $\mathrm{EV}$ pre-set by the user respectively.

- Active and reactive power constraint: When EV-ESSs respond to the control signals, their V2G capability of supplying or absorbing active and reactive power through their corresponding interfacing converters must be within the allowable range:

$$
\left\{\begin{array}{l}
-P_{i}^{\max } \leq P_{i}^{*} \leq P_{i}^{\max } \\
-Q_{i}^{\max } \leq Q_{i}^{*} \leq Q_{i}^{\max }
\end{array}\right.
$$

Where, $P_{i}^{\max }$ and $Q_{i}^{\max }$ are the maximum allowable active and reactive power respectively for $i$ th $\mathrm{EV} . P_{i}^{*}$ and $Q_{i}^{*}$ are the active and reactive power reference for the power controller illustrated in Fig. 2. The negative $(-)$ sign represents the absorption of active or reactive power by the EV-ESS.

\section{Case Studies}

In this section, simulations have been carried out to demonstrate the performance of the coordinated control strategy for three-phase (3P) and DC EV-ESS in a hybrid AC/DC microgrid with developed interlinking/interfacing controller. The hybrid AC/DC microgrid is composed of $250 \mathrm{~kW}$ PV and $60 \mathrm{kWh}$ BESS at the DC side and $48 \mathrm{kWh}$ BESS at the AC side. EV-ESS for both DC and three-phase AC configurations are $48 \mathrm{kWh}$, whereas for single-phase configuration they are $19.2 \mathrm{kWh}$. All the necessary parameters have been provided in the Appendix A. The hybrid AC/DC microgrid and the control structure have been designed in MATLAB/SIMULINK ${ }^{\circledR}$ environment using SimPowerSystems ${ }^{\mathrm{TM}}$ toolbox. The simulations are run on Intel(R) Core(TM)i5-4570 CPU 3.20 GHz with 8.00 GB RAM. Four case studies have been carried out in this paper. In case $\mathrm{A}$, the performance of the interlinking/interfacing converter controller has been demonstrated for islanded hybrid AC/DC microgrid operation considering simultaneous variable irradiation of the PV system and the commercial load profile for one day (24 hours) of N44 building without any participation of EVs. In Case B, single-phase EV charging has been introduced considering that all the single-phase EV chargers are allocated homogeneously among the three phases. Case $\mathrm{C}$ considers the heterogeneous allocation of single-phase EV chargers. In the last case study, which is case D, demonstrates the capability of the designed controller to synchronize with the grid when the grid is available.

\subsection{Case A: Variable irradiation and loading effects}

In this case study, the performance of hybrid AC/DC microgrid operation has been observed without any EV participation. The main purpose of this case study is to demonstrate the performance of the interlinking converter during the islanded operation of the microgrid. The solar irradiation data, PV output power and the load real and reactive power for the N44 building is collected using OSI PI data acquisition software. This software collects data from the building smart power meters. The solar irradiation, PV output power and the building load profile are shown in Fig. 4.1. The main control parameters for the interlinking/interfacing converter controller are $v_{d}$ and $v_{q}$. The references for $v_{d}$ and $v_{q}$ are generated by the combined action of the power controller/droop controller and the coordinated control algorithm. Later, references for $i_{d}$ and $i_{q}$ are generated to control the interlinking/interfacing converter. In Fig. 6 the interlinking converter control signals have been presented for normal operating condition.

\subsection{Case B: Homogeneous single-phase EV allocation}

In this case study, single-phase EV charging has been introduced. Initially homogeneous allocation of single-phase EV chargers have been considered. Homogeneous allocation of single-phase EV charging station means each phase (i.e. a, b and c) of the three-phase system is equally loaded with EV chargers. Usually for residential single-phase charging the overnight charging is suggested when the peak demand is 
(a) PV output power (kW)

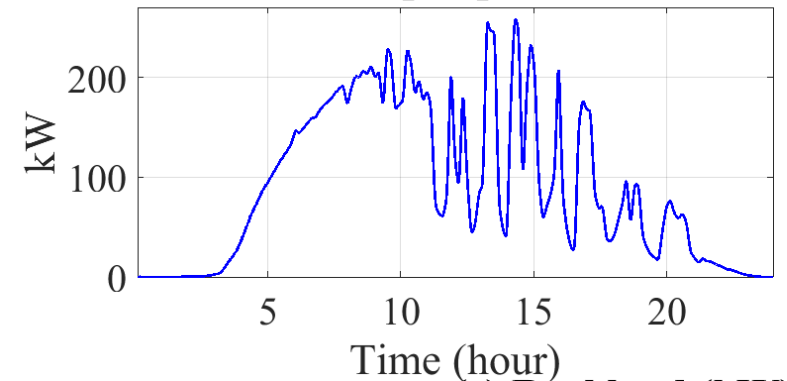

(b) Irradiation $\left(\mathbf{w} / \mathbf{m}^{2}\right)$

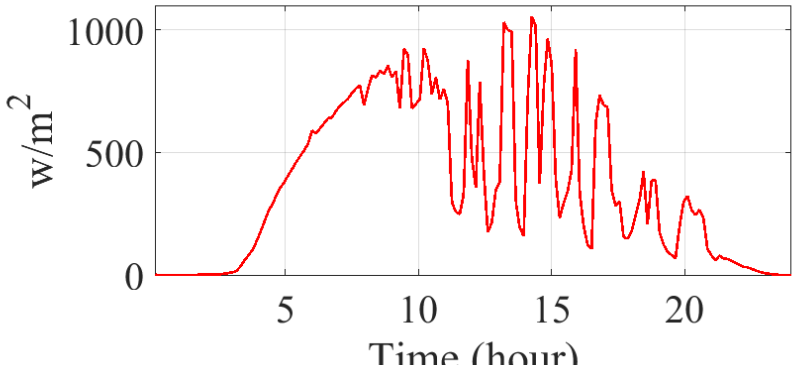

(c) Real load (kW) and Reactive load (kVAR)

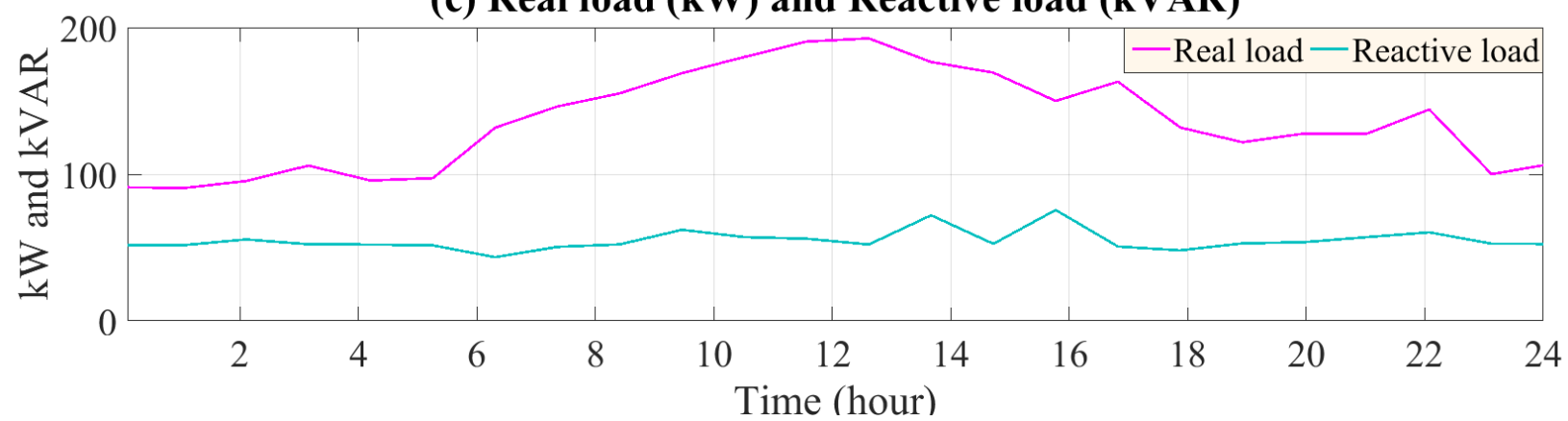

Figure 5: Input data for the hybrid AC/DC microgrid: (a) PV output power, (b) Solar irradiation and (c) Usual active and reactive power demand of the building for 24 hours
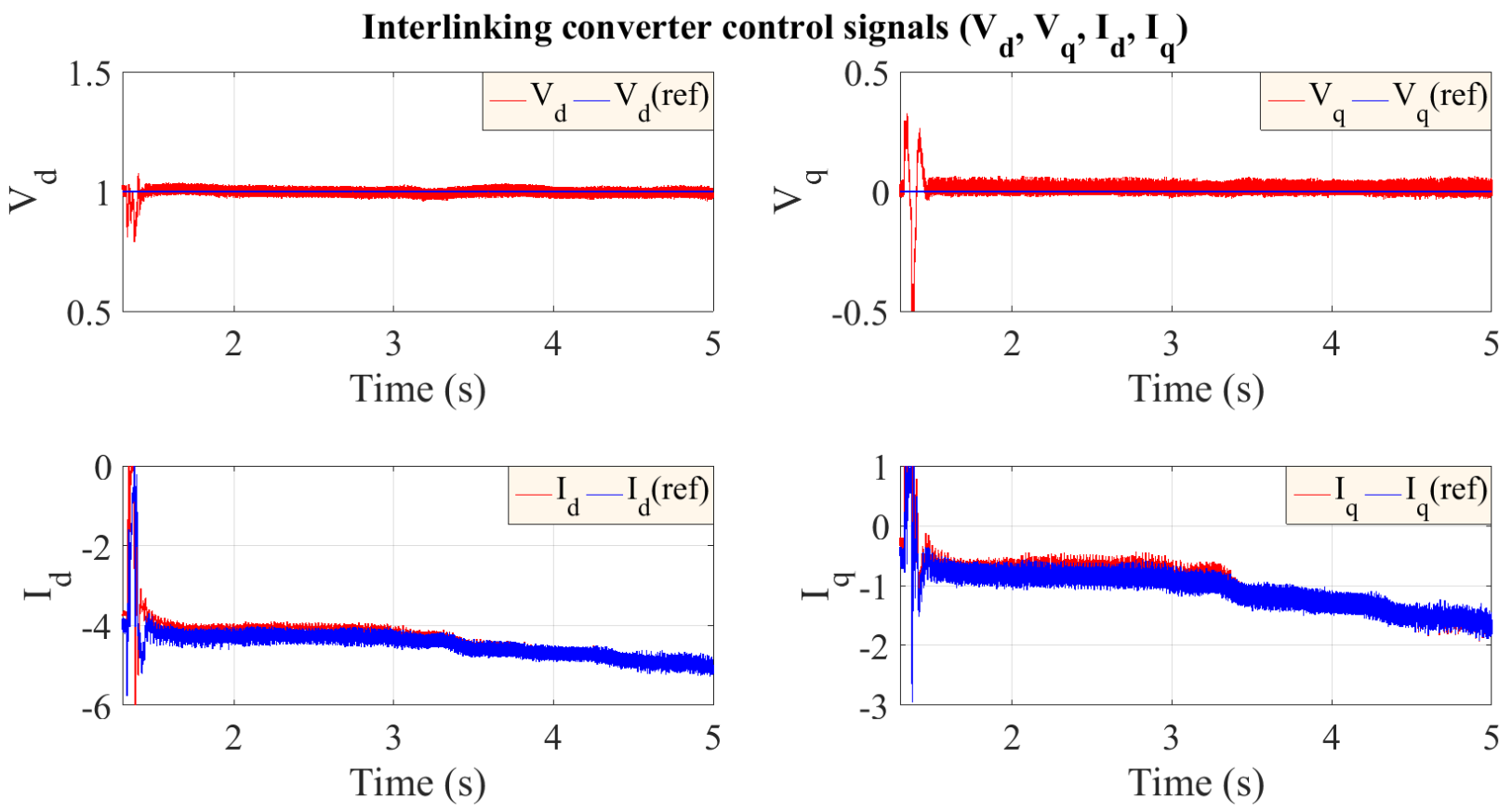

Figure 6: Control signals of the interlinking converter 


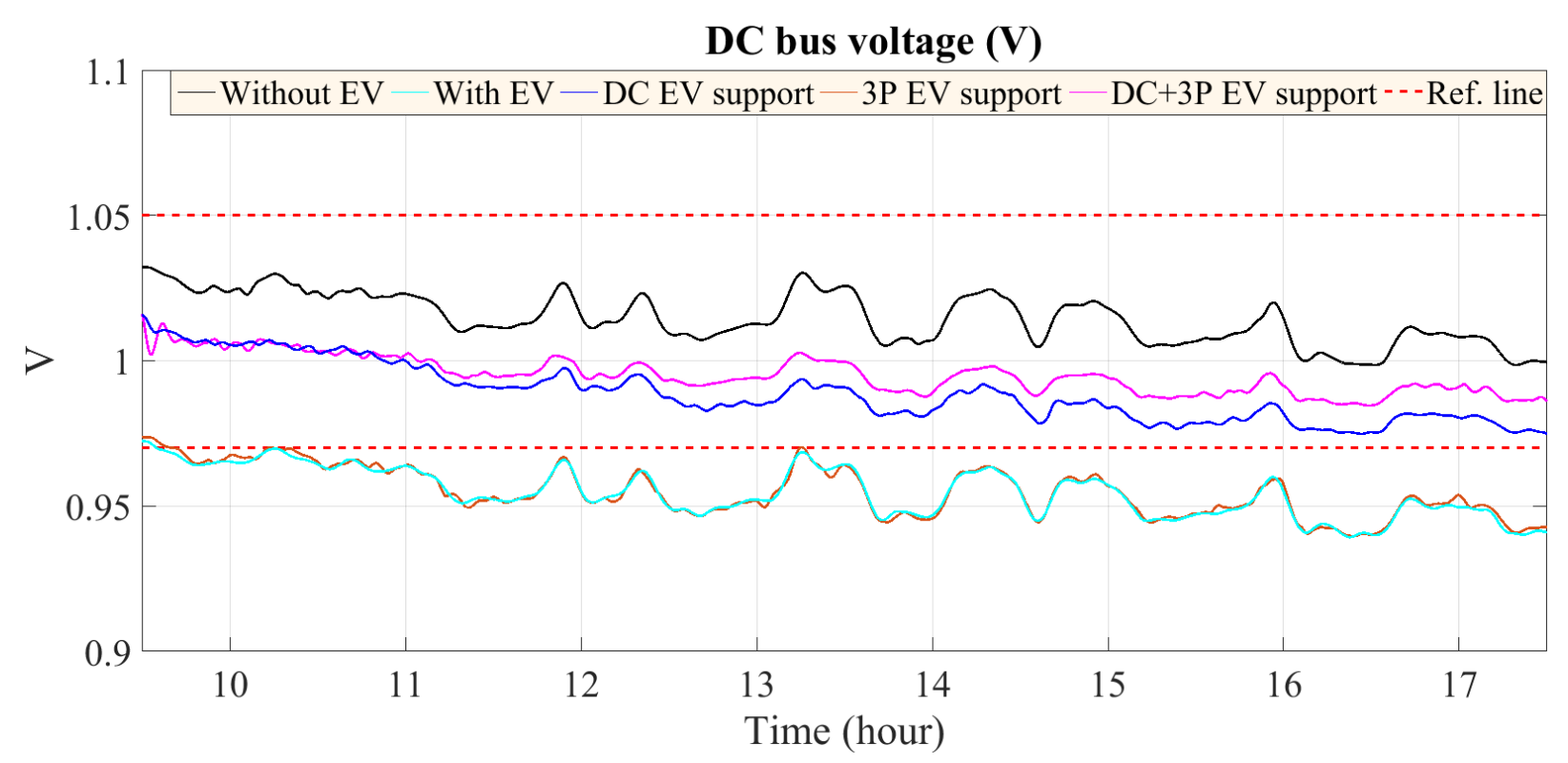

Figure 7: DC bus voltage

less and EV owners are at home. Unlike residential EV charging the commercial EV owners need to charge their EVs during day time while they are working in office or for this case in the university campus. So the charging period has been taken for this simulation is form 9:30 AM till 5:30 PM, which is usual office hours. Results obtained from this simulation for DC bus voltage, AC bus RMS voltage and frequency are presented in Fig. 7, 8 and 9 respectively. Both AC and DC bus voltages are measured in per unit (p.u.). It can be observed from the figures that the DC and AC bus voltages and system frequency are within acceptable limit during the islanded operation when there is no EVs are participating for charging. With the introduction of EV charging in each phase, there is a drop in both DC bus voltage and AC bus RMS voltage while the frequency is not severely affected due to the droop control mechanism. In order to keep the AC and DC bus voltage within acceptable limit three coordination methods have been utilized. These methods include, participation of only DC EV-ESS, participation of only 3P EV-ESS and for the worst case scenario, simultaneous participation of DC and 3P type EV-ESS operation has been considered. It can be observed that only 3P EV-ESS support is not enough to ensure DC bus voltage regulation, though it can ensure regulated AC bus RMS voltage. It is evident that simultaneous DC and 3P EV-ESS operation (which is referred as aggregated operation) improves both $\mathrm{AC}$ and DC bus voltage effectively. However, aggregated operation may not be economical for long time operation. Comparative simulation results for DC bus voltage, AC bus RMS voltage and frequency considering without EVs, with EVs, only DC EV-ESS support, only 3P EV-ESS support and aggregated operation are shown in Fig. 7, 8 and 9 respectively. Meanwhile, the state-of-charge (SOC) for charging and discharging EV-ESS considering all these modes are shown in Fig. 10 and 11. Active and reactive power profiles for the aggregated operation are shown in Fig. 12 and 13 to show the effectiveness of the designed droop controller.

\subsection{Case C: Heterogeneous single-phase EV allocation}

Heterogeneous allocation EV-ESS has been considered for this case study. It refers that each phase in loaded whimsically as presented in Fig. 14. It should be noted that each single-phase EV chargers has been designed to act as active load operation. The active load operation of EV is designed with single-phase PLL and corresponding controllers as presented in [45]. It can be observed from the Fig. 15 and 16 that even with the unbalanced loading condition the controller effectively regulate DC and AC bus voltages and frequency. 


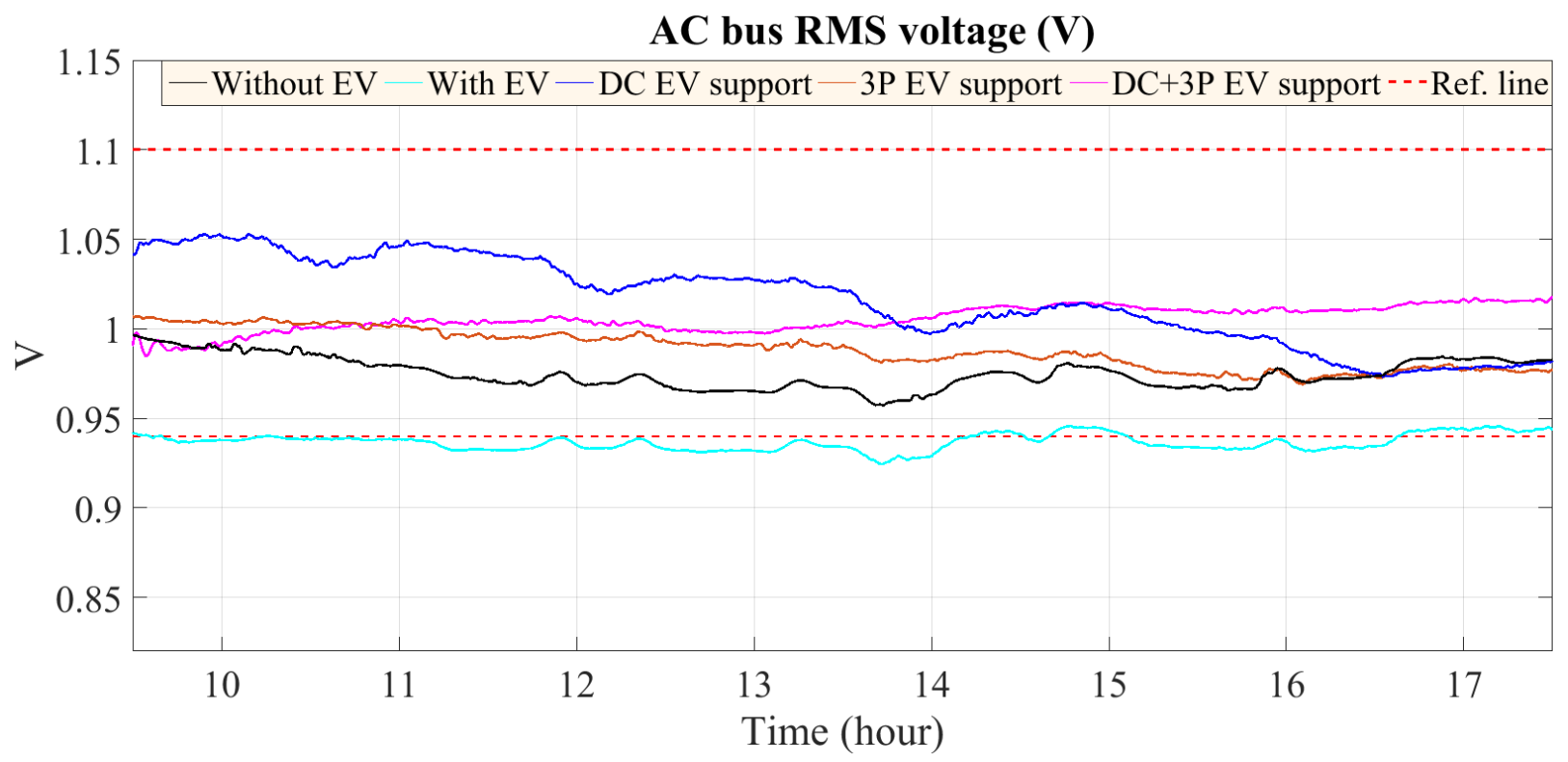

Figure 8: AC bus RMS voltage (line to neutral)

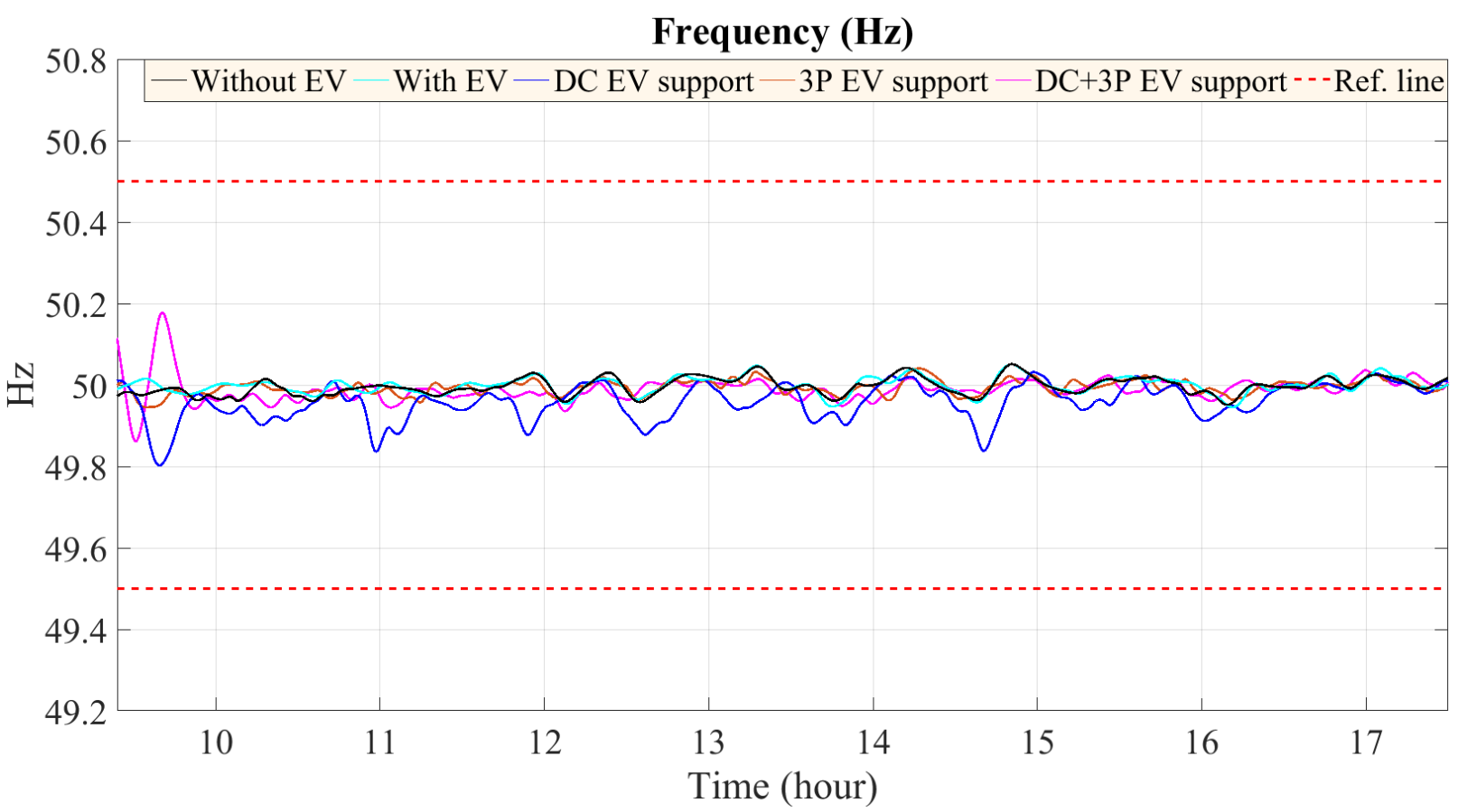

Figure 9: Frequency 

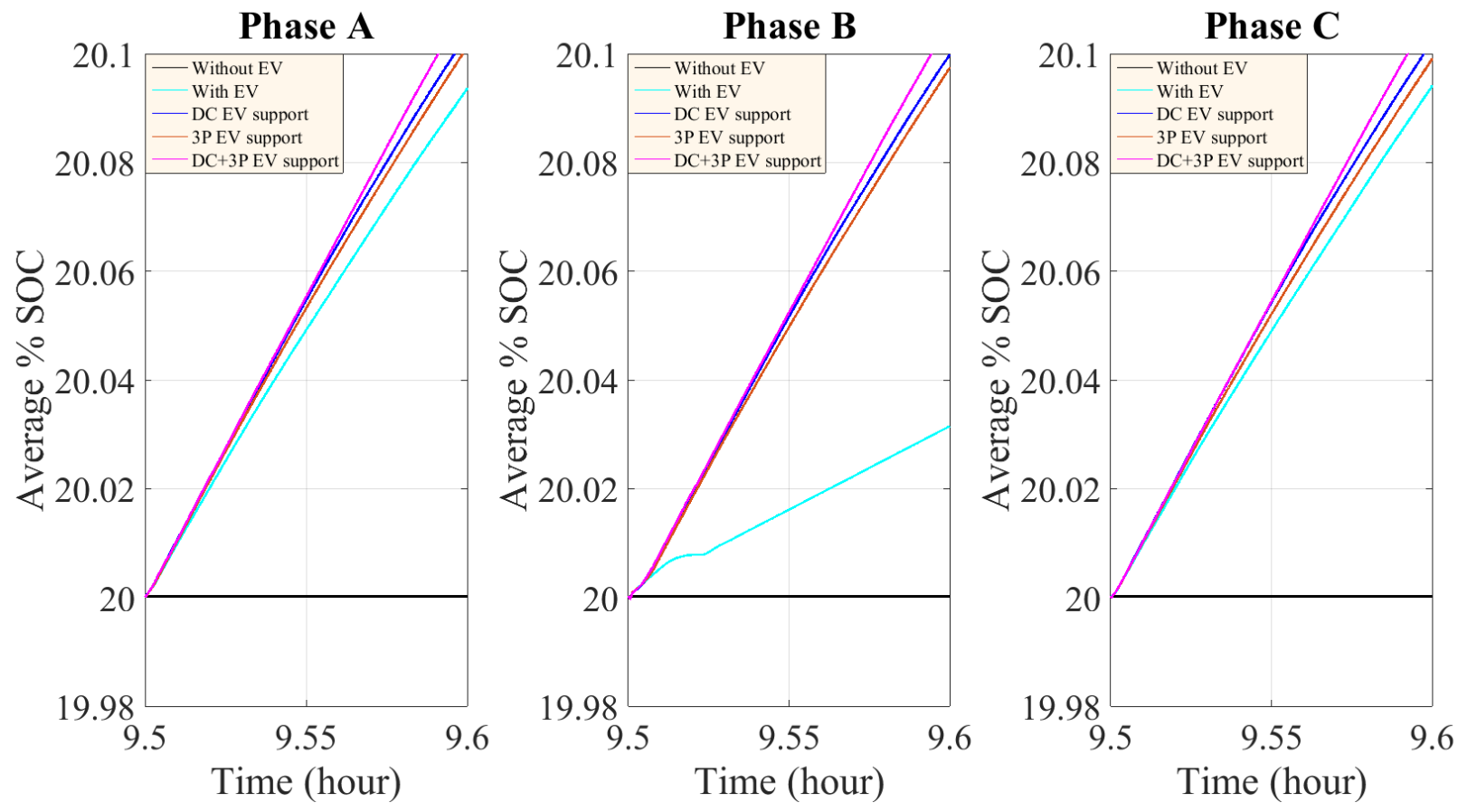

Figure 10: SOC of the single-phase EV-ESS
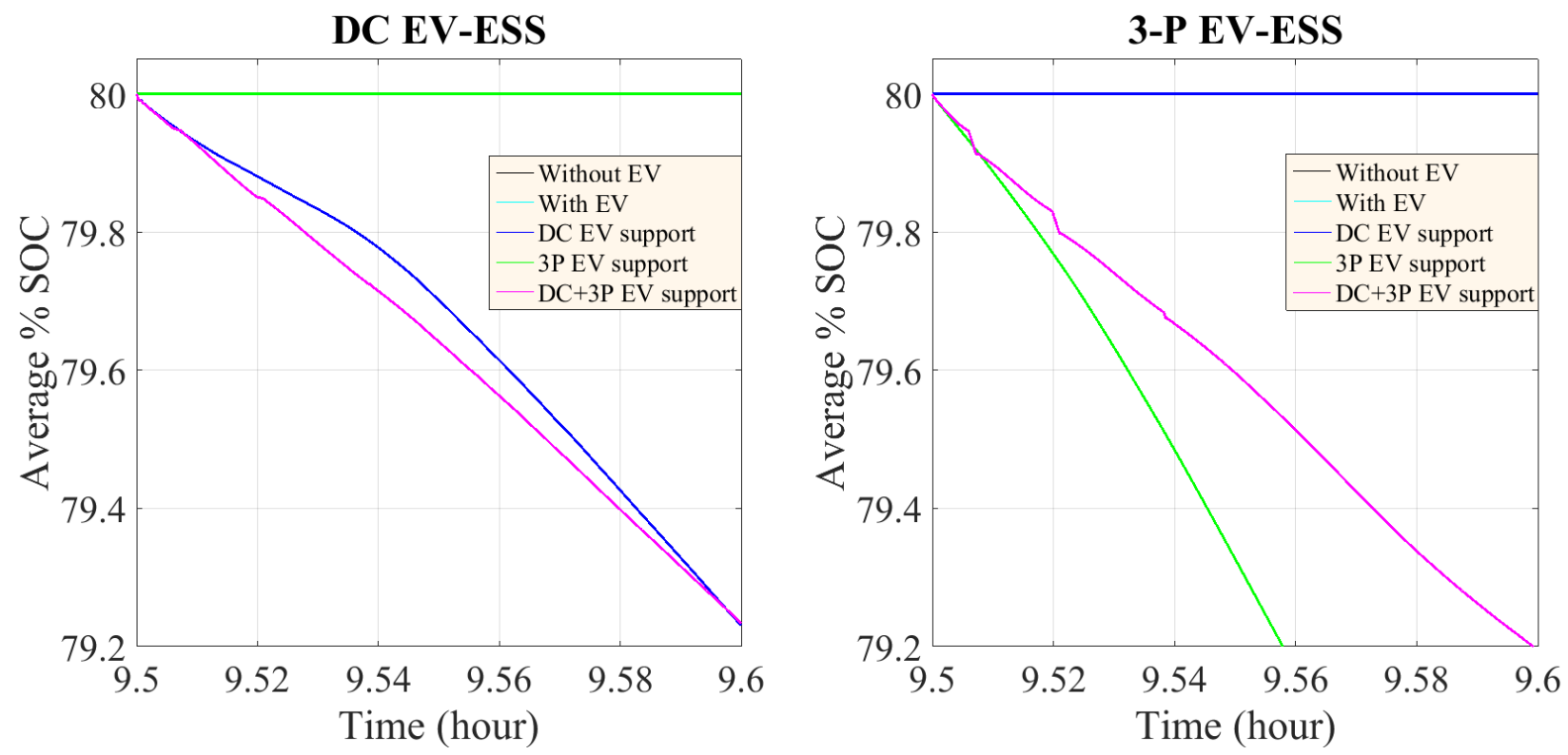

Figure 11: SOC of the DC type and 3P type EV-ESS 


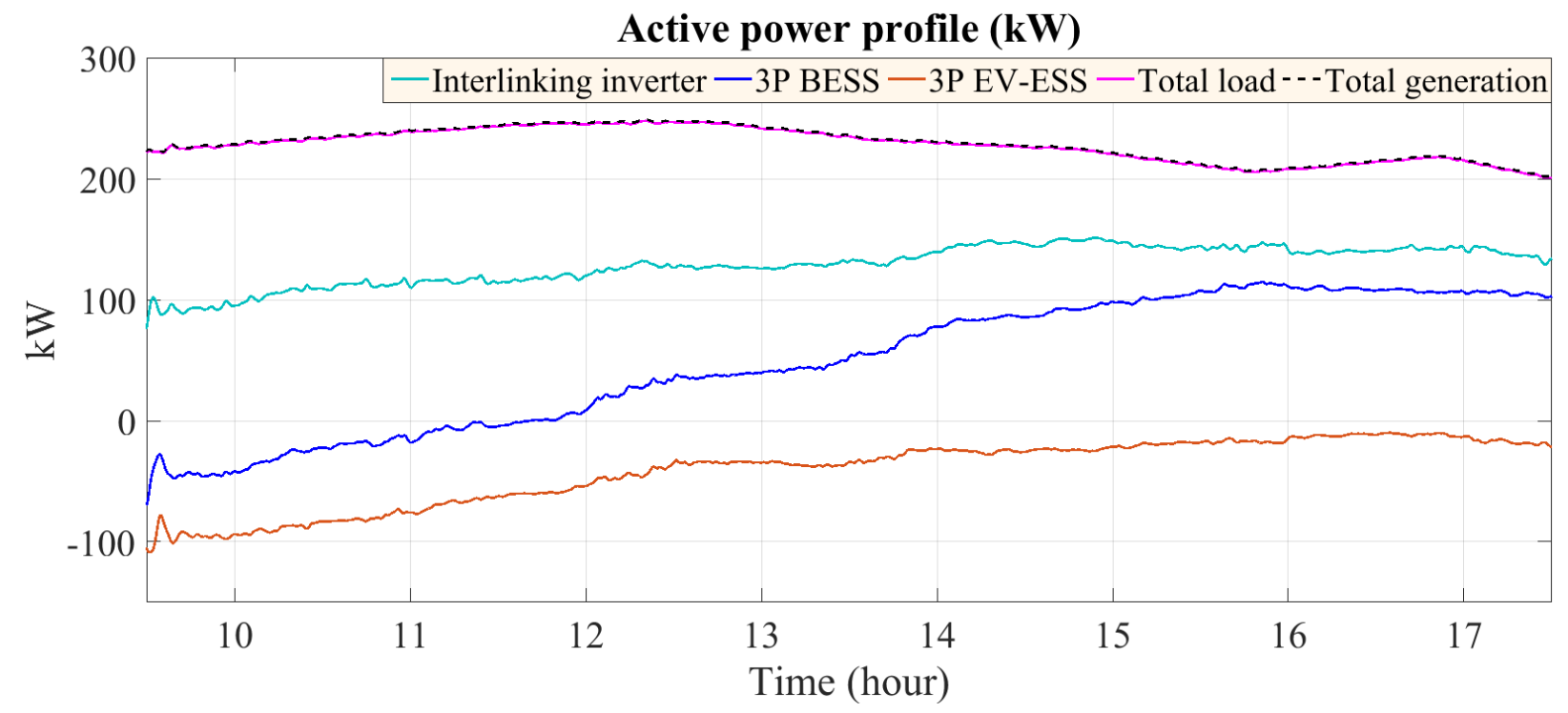

Figure 12: Active power profile of the system

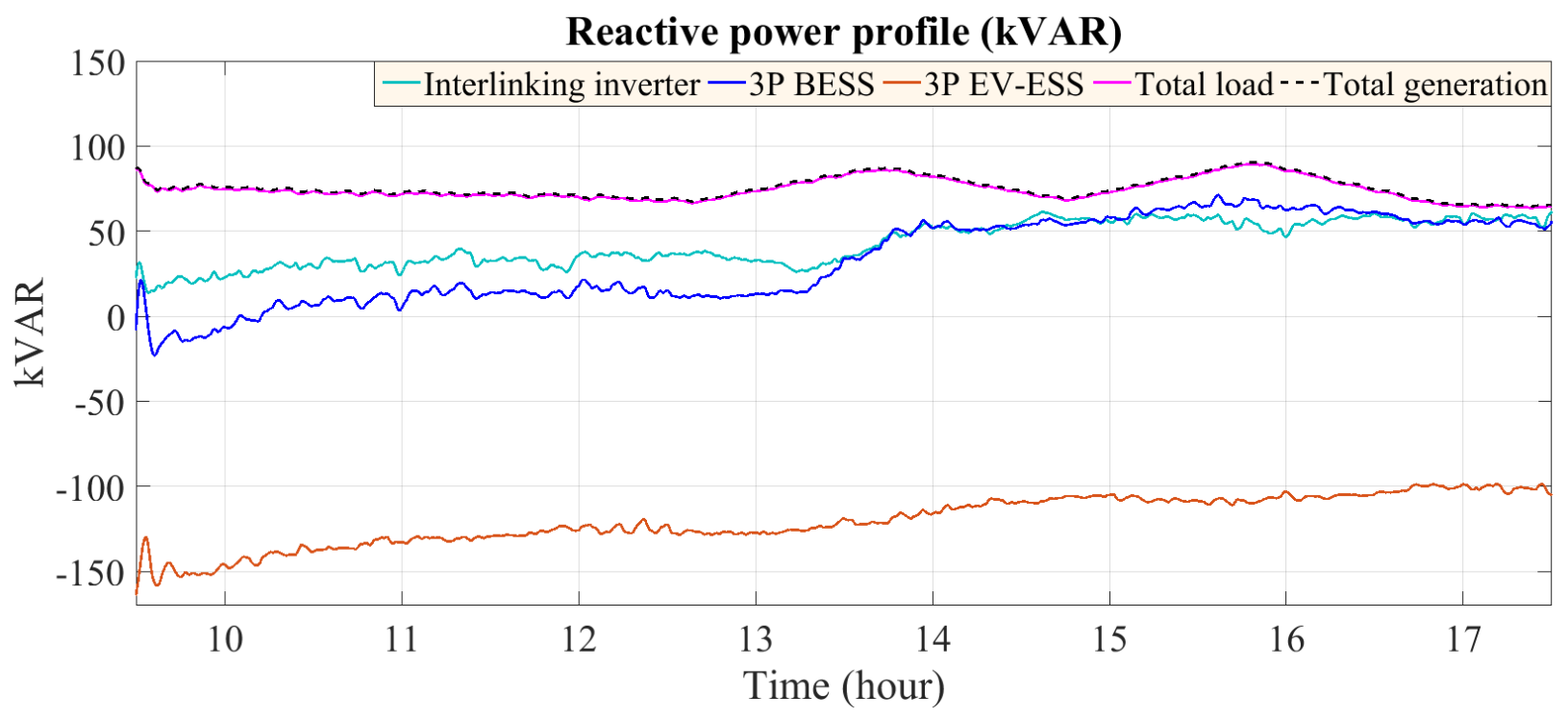

Figure 13: Reactive power profile of the system 
(a) Single phase EV power (kW)

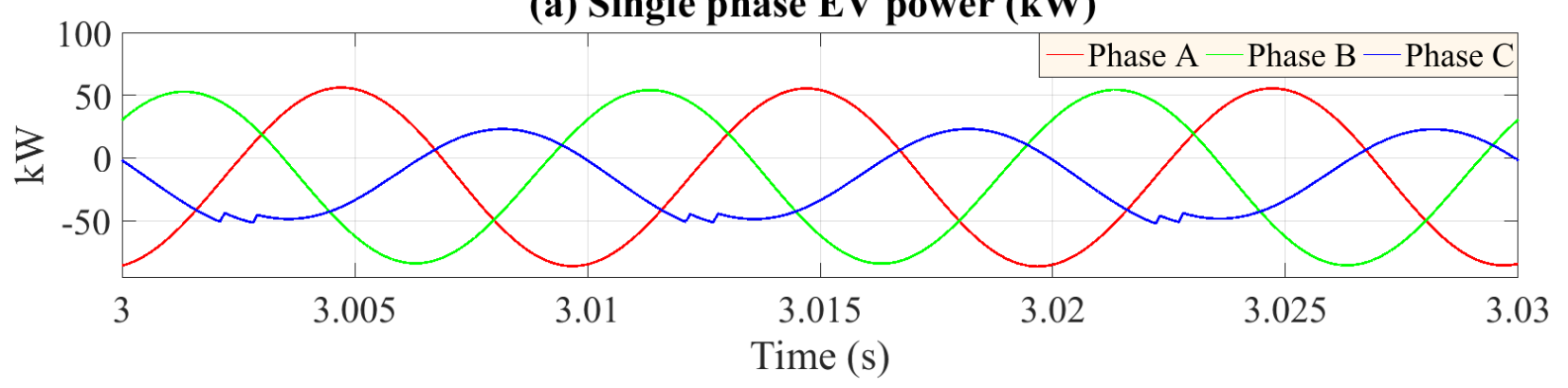

(b) Single phase EV RMS power (kW)

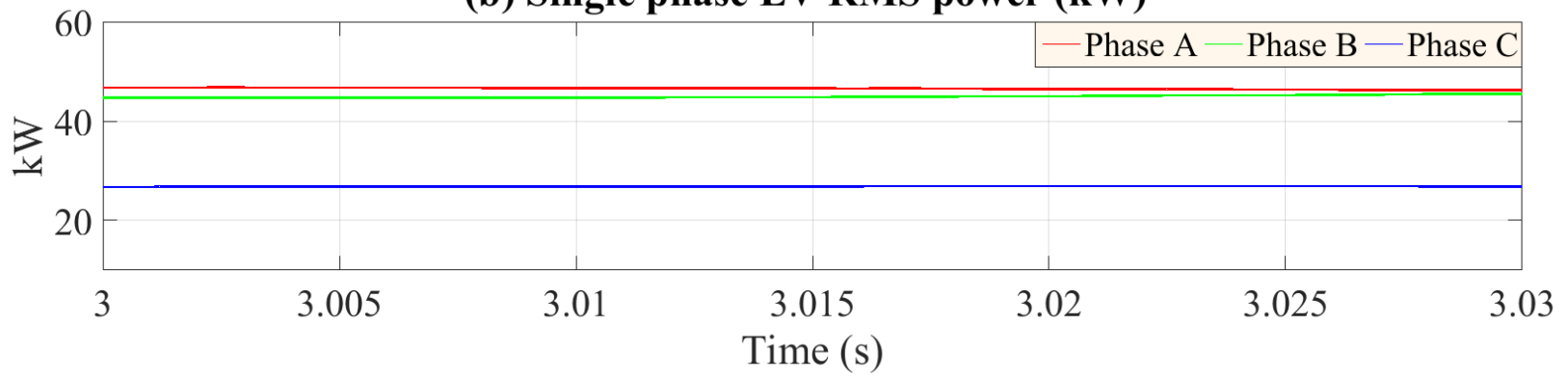

Figure 14: Charging power drawn by single-phase EV-ESS: (a) Single-phase power and (b) RMS value of the single -phase power
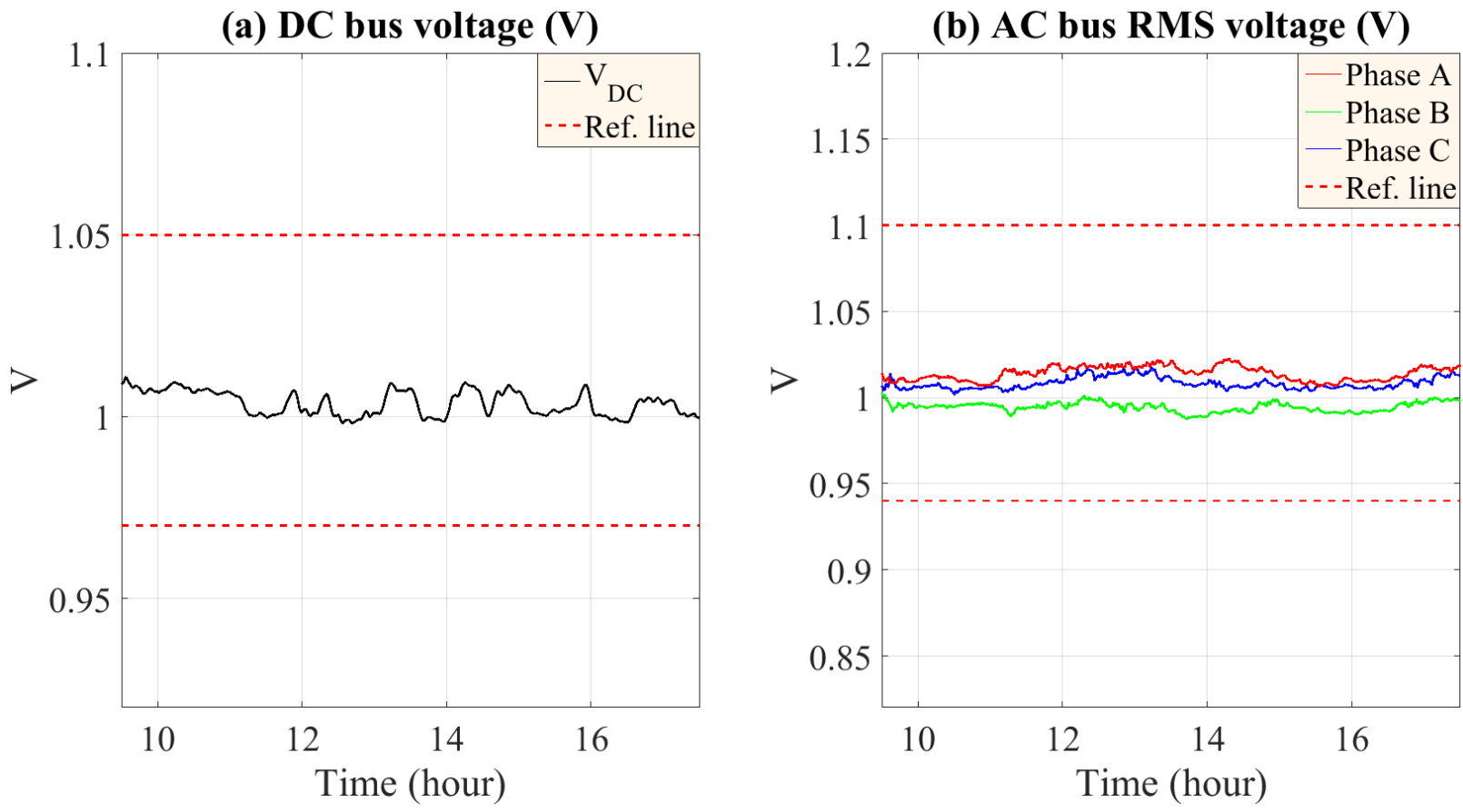

Figure 15: Voltage profile: (a) DC bus voltage and (b) AC bus RMS voltage 


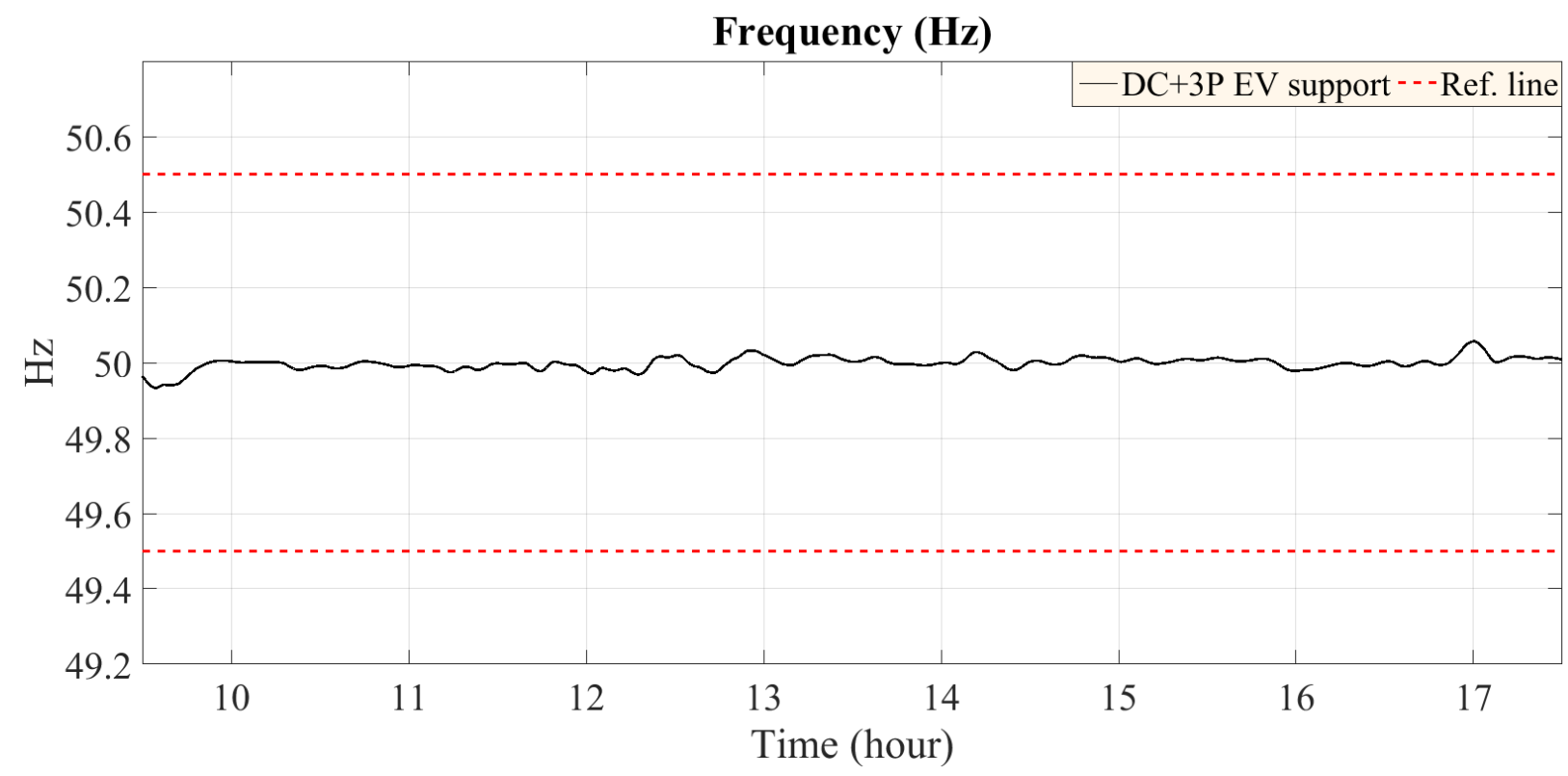

Figure 16: Frequency

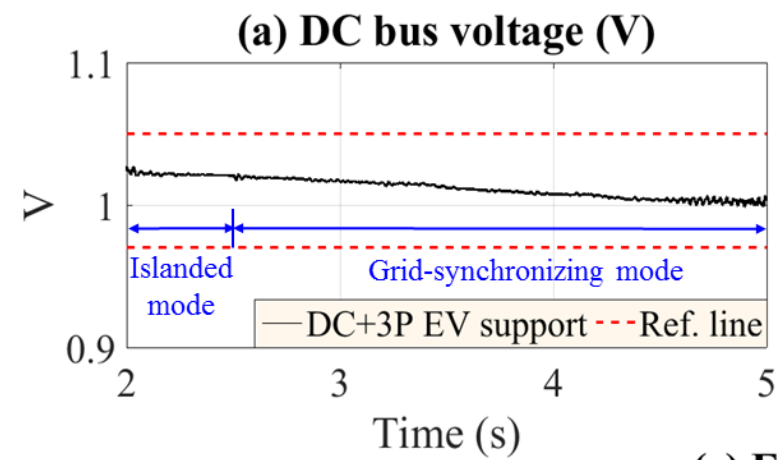

(c) Frequency (Hz)

(b) AC bus RMS voltage (V)

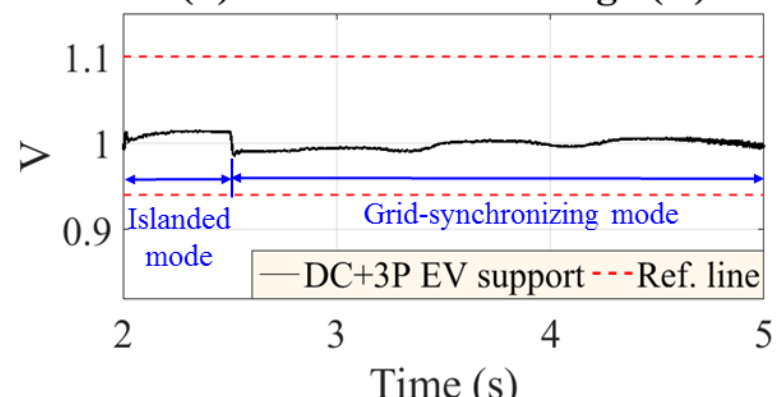

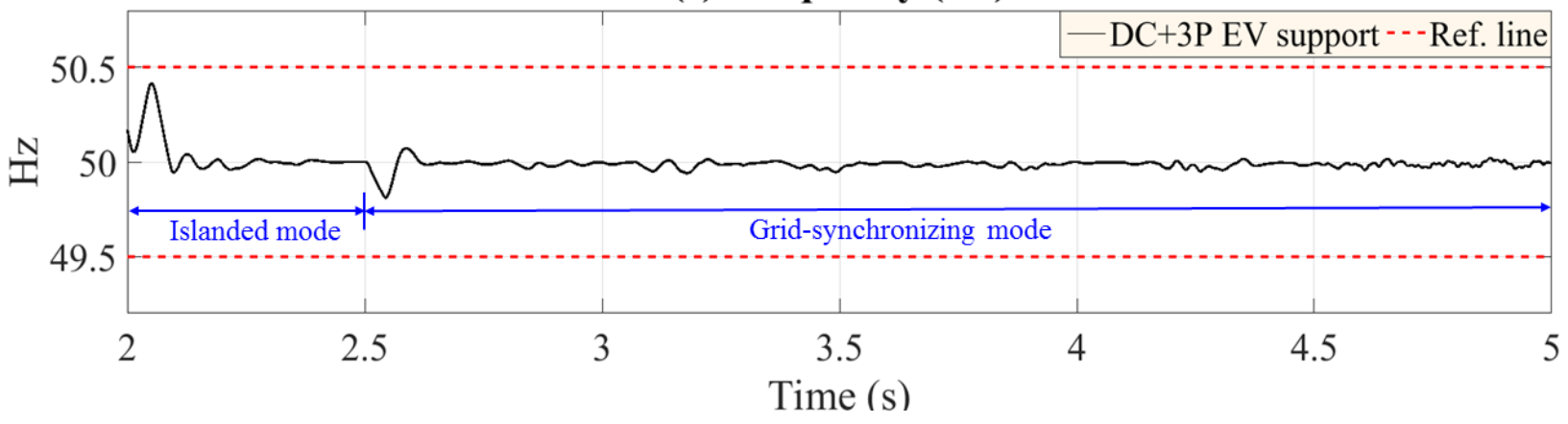

Figure 17: Grid synchronization mode: (a) DC bus voltage, (b) AC bus RMS voltage and (c) Frequency 


\subsection{Case D: Grid synchronization}

Meanwhile, microgrid may need to switch from islanded to grid connected condition during contingency. During the switching period from islanded mode to grid connected mode, the designed controller ensures smooth operation and synchronization with the main grid. In order to connect from the islanded to grid connected mode, the magnitude and the frequency of the AC side PCC voltage has to be in synchronism with the grid voltage magnitude and frequency. From the case study results presented in Fig. 17, it can be observed that DC bus voltage, AC bus RMS voltage and frequency remain in synchronism even in the transition period from islanded to grid connected mode.

\section{Conclusion}

In this paper, a novel coordinated control strategy is proposed which facilitates the V2G capability of three-phase and DC type EV charging stations in an islanded commercial hybrid AC/DC microgrid. The main objective of the coordinated control is to improve the performance of the microgrid in terms of voltage and frequency regulation, active/reactive power management and unbalanced condition. Realistic EV availability constraints are considered for the design process. Three concurrently operating and nonoverlapping layers of coordination has been developed. The first layer manages the DC subgrid of the hybrid AC/DC microgrid and ensures stiff DC bus voltage regulation by coordinating DC type EV chargers. The second and third layer coordinate three-phase EV charging station that has the V2G four quadrant operational capability which are responsible for frequency and AC bus RMS voltage regulation respectively. As a single entity, the coordination algorithm is embedded into the microgrid central controller (MGCC) which generates necessary active/reactive power command for microgrid local controllers such as interlinking and interfacing controllers. The designed controller with proposed algorithm has been tested considering PV with fluctuating output and random commercial loads. Based on the comparative simulation results, improved voltage regulation and power sharing performance have been observed even with the presence of homogeneous and heterogeneous single-phase EV charging. The future vision for this research is to integrate the market operation and to investigate the effects of the distributed control approach to reduce communication dependency.

\section{Acknowledgement}

This work is partially supported by the Queensland Government-funded Smart Future Partnership Project and the doctoral scholarship offered by the Griffith University. 


\section{Appendix A. Simulation parameters}

Table A.2: Simulation parameters for the hybrid AC/DC microgrid

\begin{tabular}{|c|c|c|c|c|c|c|c|}
\hline \multicolumn{8}{|c|}{ Energy nodes } \\
\hline \multicolumn{4}{|c|}{ Solar PV } & \multicolumn{4}{|c|}{$250 \mathrm{~kW}$} \\
\hline \multicolumn{4}{|c|}{ PV BESS } & \multicolumn{4}{|c|}{$60 \mathrm{kWh}$} \\
\hline \multicolumn{4}{|c|}{ Three-phase BESS } & \multicolumn{4}{|c|}{$48 \mathrm{kWh}$} \\
\hline \multicolumn{8}{|c|}{ EV-ESS } \\
\hline \multicolumn{4}{|c|}{ DC EV-ESS } & \multicolumn{4}{|c|}{$48 \mathrm{kWh}$} \\
\hline & \multicolumn{4}{|c|}{$48 \mathrm{kWh}$} \\
\hline \multicolumn{4}{|c|}{ Single-phase EV-ESS } & \multicolumn{4}{|c|}{$19.2 \mathrm{kWh}$} \\
\hline \multicolumn{8}{|c|}{ Line parameters } \\
\hline \multicolumn{4}{|c|}{ Nominal AC bus RMS voltage (line to neutral) } & \multicolumn{4}{|c|}{$230 \sim 240 \mathrm{~V}$} \\
\hline \multicolumn{4}{|c|}{ Nominal DC bus voltage } & \multicolumn{4}{|c|}{$600 \sim 800 \mathrm{~V}$} \\
\hline \multicolumn{4}{|c|}{ Frequency } & \multicolumn{4}{|c|}{$50 \mathrm{~Hz}$} \\
\hline \multicolumn{4}{|c|}{$C_{D C}$} & \multicolumn{4}{|c|}{$100 \times 10^{-3} \mathrm{~F}$} \\
\hline \multicolumn{4}{|l|}{$R_{f}$} & \multicolumn{4}{|c|}{$2 \times 10^{-3} \Omega$} \\
\hline \multicolumn{4}{|l|}{$L_{f}$} & \\
\hline \multicolumn{4}{|l|}{$C_{f}$} & \multicolumn{3}{|c|}{$250 \times 10^{-6} H$} & $16.9 \times 10^{-3} F$ \\
\hline \multicolumn{4}{|l|}{$R_{c}$} & & $2 \times 1$ & $j^{-3} \Omega$ & \\
\hline$L_{c}$ & & & & & $500 \times$ & $0^{-6} H$ & \\
\hline $\mathrm{Dam}_{1}$ & ing resistor & & & & $20 \times$ & $0^{-2} \Omega$ & \\
\hline & & & Control para & neters & & & \\
\hline Pov & er controller & Vol & age controller & Curre & t controller & $\mathrm{DC} / \mathrm{D}$ & converter \\
\hline$\omega_{c}$ & $31.42 \mathrm{rad} / \mathrm{s}$ & & 4.1497 & $K_{p(I)}$ & 0.7390 & & 7 \\
\hline$V^{*}$ & $340 \mathrm{~V}$ & $K_{i(V)}$ & $3.90 \times 10^{3}$ & $K_{i(I)}$ & $3.17 \times 10^{4}$ & $\begin{array}{c}p(V) \\
K_{i(V)}^{D C}\end{array}$ & 800 \\
\hline$\omega^{*}$ & $314.16 \mathrm{rad} / \mathrm{s}$ & $\mathrm{F}$ & 0.75 & & & $K_{p(I)}^{D C}$ & 0.3 \\
\hline$D_{P}$ & $3.14 \times 10^{-5}$ & & & & & $\begin{array}{c}p(1 \\
K_{i(I)}^{D C}\end{array}$ & 20 \\
\hline$D_{Q}$ & $1.36 \times 10^{-4}$ & & & & & & \\
\hline$K_{p(P)}$ & $2.5 \times 10^{-4}$ & & & & & & \\
\hline $\begin{array}{l}n_{p(P)} \\
K_{d(P)}\end{array}$ & $3 \times 10^{-3}$ & & & & & & \\
\hline
\end{tabular}




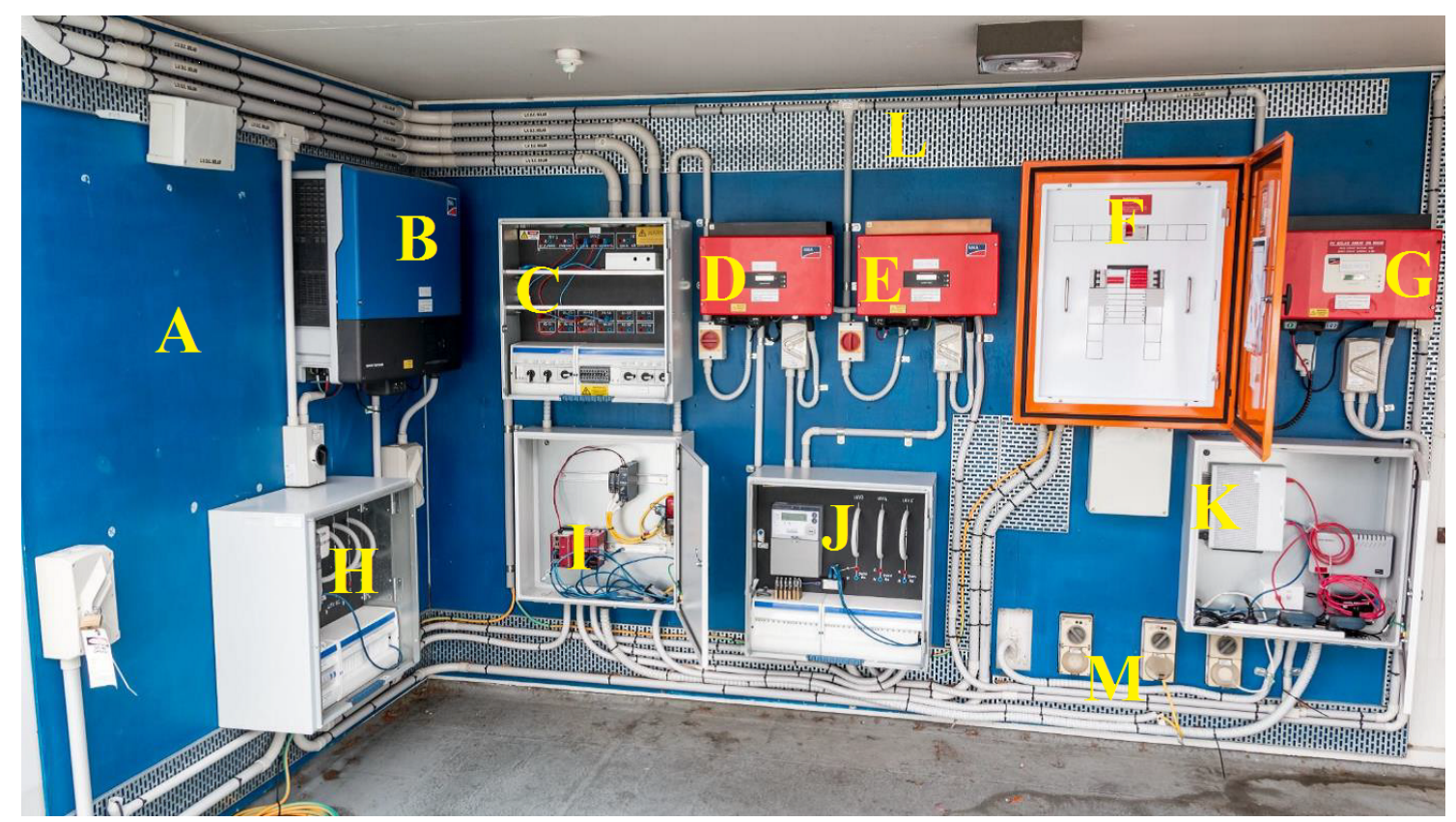

Figure B.18: Microrgrid components in N44 building

Table B.3: N44 microgrid testing facility equipment

\begin{tabular}{cl}
\hline Identifier & \multicolumn{1}{c}{ Description } \\
\hline A & Allocated location for D-STATCOM \\
B & 10 kW SMA inverter \\
C & DC marshalling, micro-inverter and DC/DC converter enclosure \\
D & 2 kW SMA Inverter \\
E & 160 A distribution board \\
F & 1.5 kW SMA inverter \\
G & 3-phase monitoring enclosure \\
H & 3-phase monitoring and protection unit \\
I & Red-lion communication adapter \\
J & Single-phase monitoring enclosure \\
K & Data connection point with SMA Webbox \\
L & DC bus \\
M & AC bus \\
\hline
\end{tabular}




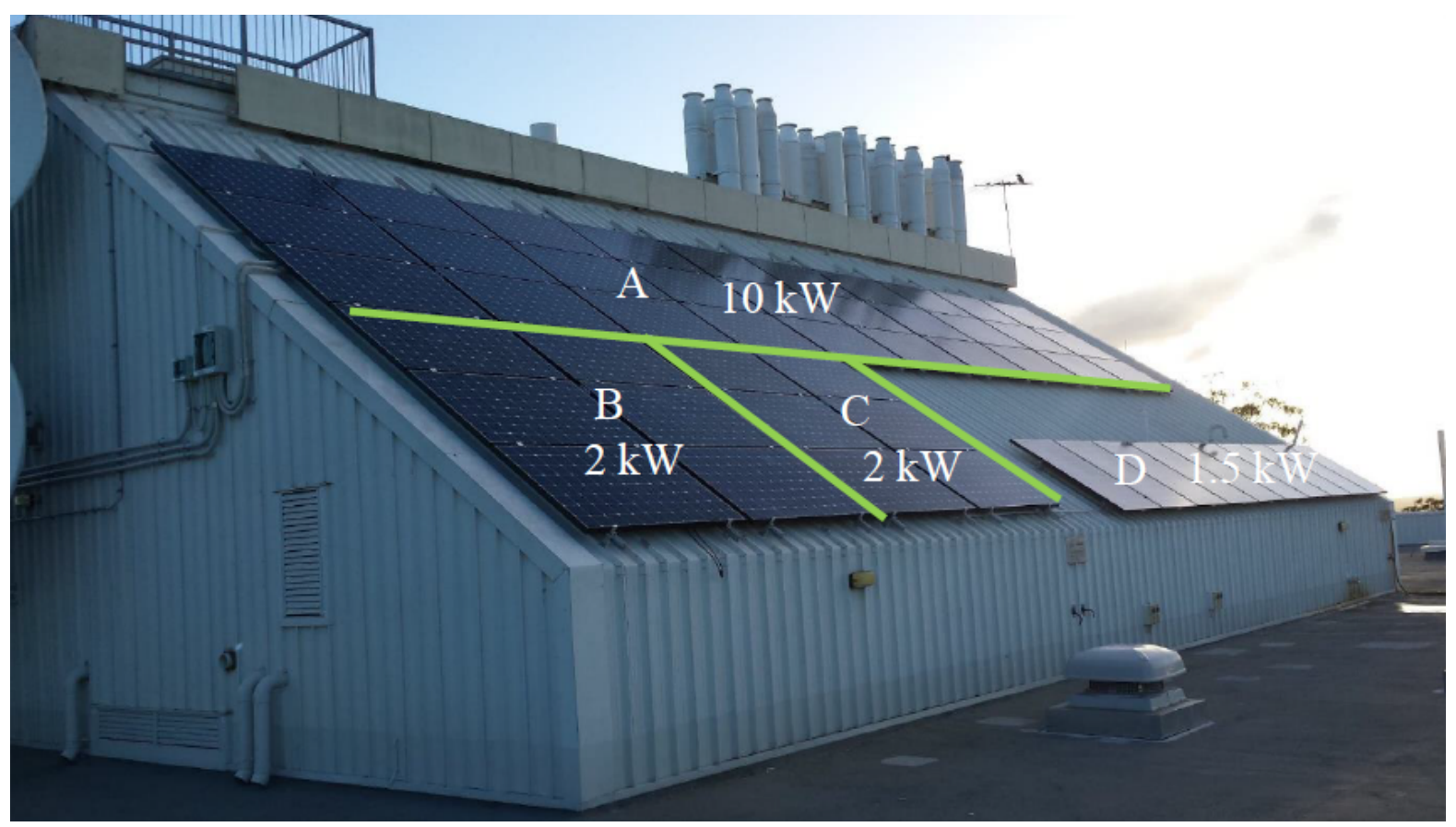

Figure B.19: Rooftop PV installation on N44 building

Table B.4: Installed solar PV specification

\begin{tabular}{cl}
\hline Identifier & \multicolumn{1}{c}{ Description } \\
\hline $\mathrm{A}$ & $10 \mathrm{~kW}$ peak SunPower E20 series solar panels in 6 strings of 5 panels \\
$\mathrm{B}$ & $2 \mathrm{~kW}$ peak SunPower E20 series solar panels \\
$\mathrm{C}$ & $2 \mathrm{~kW}$ peak SunPower E20 series solar panels \\
$\mathrm{D}$ & $1.5 \mathrm{~kW}$ peak Kyocera modules in series configuration \\
\hline
\end{tabular}

\section{References}

[1] E. Hossain, E. Kabalci, R. Bayindir, and R. Perez, "Microgrid testbeds around the world: state of art," Energy Conversion and Management, vol. 86, pp. 132-153, 2014, ISSN: 0196-8904. DOI: http://dx.doi.org/10.1016/j.enconman.2014.05. 012.

[2] X. Liu, P. Wang, and P. C. Loh, "A hybrid ac/dc microgrid and its coordination control," IEEE Transactions on Smart Grid, vol. 2, no. 2, pp. 278-286, Jun. 2011, ISSN: 1949-3053. DOI: 10.1109/TSG.2011.2116162.

[3] P. C. Loh, D. Li, Y. K. Chai, and F. Blaabjerg, "Autonomous control of interlinking converter with energy storage in hybrid ac x2013; dc microgrid," IEEE Transactions on Industry Applications, vol. 49, no. 3, pp. 1374-1382, May 2013, ISSN: 0093-9994. DOI: 10.1109/TIA.2013.2252319.

[4] J. M. Guerrero, P. C. Loh, T. L. Lee, and M. Chandorkar, "Advanced control architectures for intelligent microgrids x2014;part ii: power quality, energy storage, and ac/dc microgrids," IEEE Transactions on Industrial Electronics, vol. 60, no. 4, pp. 1263-1270, Apr. 2013, ISSN: 0278-0046. DOI: 10.1109/TIE.2012.2196889.

[5] F. Nejabatkhah and Y. W. Li, "Overview of power management strategies of hybrid ac x002f;dc microgrid," IEEE Transactions on Power Electronics, vol. 30, no. 12, pp. 7072-7089, Dec. 2015, ISSN: 0885-8993. DOI: 10.1109/TPEL. 2014. 2384999.

[6] J. V. Roy, N. Leemput, F. Geth, J. Büscher, R. Salenbien, and J. Driesen, "Electric vehicle charging in an office building microgrid with distributed energy resources," IEEE Transactions on Sustainable Energy, vol. 5, no. 4, pp. 1389-1396, Oct. 2014, ISSN: 1949-3029. DOI: 10.1109/TSTE.2014.2314754.

[7] N. Liu, Q. Chen, J. Liu, X. Lu, P. Li, J. Lei, and J. Zhang, "A heuristic operation strategy for commercial building microgrids containing evs and pv system," IEEE Transactions on Industrial Electronics, vol. 62, no. 4, pp. 2560-2570, Apr. 2015, ISSN: 0278-0046. DOI: 10.1109/TIE. 2014.2364553. 
[8] S. Beer, T. Gomez, D. Dallinger, I. Momber, C. Marnay, M. Stadler, and J. Lai, "An economic analysis of used electric vehicle batteries integrated into commercial building microgrids," IEEE Transactions on Smart Grid, vol. 3, no. 1, pp. 517-525, Mar. 2012, ISSN: 1949-3053. DOI: 10.1109/TSG.2011.2163091.

[9] C. Guille and G. Gross, "A conceptual framework for the vehicle-to-grid (v2g) implementation," Energy Policy, vol. 37, no. 11, pp. 4379-4390, 2009, ISSN: 0301-4215. DOI: http://dx.doi.org/10.1016/j.enpol.2009.05.053.

[10] M. Honarmand, A. Zakariazadeh, and S. Jadid, "Integrated scheduling of renewable generation and electric vehicles parking lot in a smart microgrid," Energy Conversion and Management, vol. 86, pp. 745-755, 2014, ISSN: 01968904. DOI: 10.1016/j.enconman.2014.06.044.

[11] A. Zakariazadeh, S. Jadid, and P. Siano, "Integrated operation of electric vehicles and renewable generation in a smart distribution system," Energy Conversion and Management, vol. 89, pp. 99-110, 2015, ISSN: 01968904. DOI: 10.1016/j . enconman.2014.09.062.

[12] N. Eghtedarpour and E. Farjah, "Power control and management in a hybrid ac/dc microgrid," IEEE Transactions on Smart Grid, vol. 5, no. 3, pp. 1494-1505, May 2014, ISSN: 1949-3053. DOI: 10.1109/TSG.2013.2294275.

[13] M. Hosseinzadeh and F. R. Salmasi, "Robust optimal power management system for a hybrid ac/dc micro-grid," IEEE Transactions on Sustainable Energy, vol. 6, no. 3, pp. 675-687, Jul. 2015, ISSN: 1949-3029. DOI: 10.1109/TSTE.2015. 2405935.

[14] M. C. Kisacikoglu, B. Ozpineci, and L. M. Tolbert, "Ev/phev bidirectional charger assessment for v2g reactive power operation," IEEE Transactions on Power Electronics, vol. 28, no. 12, pp. 5717-5727, Dec. 2013, ISSN: 0885-8993. DOI: 10.1109/TPEL. 2013.2251007.

[15] M. Kesler, M. C. Kisacikoglu, and L. M. Tolbert, "Vehicle-to-grid reactive power operation using plug-in electric vehicle bidirectional offboard charger," IEEE Transactions on Industrial Electronics, vol. 61, no. 12, pp. 6778-6784, Dec. 2014, ISSN: 0278-0046. DOI: 10.1109/TIE. 2014.2314065.

[16] P. Mitra, G. K. Venayagamoorthy, and K. A. Corzine, "Smartpark as a virtual statcom," IEEE Transactions on Smart Grid, vol. 2, no. 3, pp. 445-455, Sep. 2011, ISSN: 1949-3053. DOI: 10.1109/TSG.2011.2158330.

[17] J. Zhong, L. He, C. Li, Y. Cao, J. Wang, B. Fang, L. Zeng, and G. Xiao, "Coordinated control for large-scale \{ev\} charging facilities and energy storage devices participating in frequency regulation," Applied Energy, vol. 123, pp. 253-262, 2014, ISSN: 0306-2619. DOI: http://dx.doi.org/10.1016/j.apenergy.2014.02.074.

[18] C. Gouveia, C. L. Moreira, J. A. P. Lopes, D. Varajao, and R. E. Araujo, "Microgrid service restoration: the role of plugged-in electric vehicles," IEEE Industrial Electronics Magazine, vol. 7, no. 4, pp. 26-41, Dec. 2013, ISSN: 1932-4529. DOI: $10.1109 /$ MIE. 2013.2272337.

[19] M. F. M. Arani, Y. A. R. I. Mohamed, and E. F. El-Saadany, "Analysis and mitigation of the impacts of asymmetrical virtual inertia," IEEE Transactions on Power Systems, vol. 29, no. 6, pp. 2862-2874, Nov. 2014, ISSN: 0885-8950. DOI: 10.1109/TPWRS. 2014.2309675.

[20] S. Weckx and J. Driesen, "Load balancing with ev chargers and pv inverters in unbalanced distribution grids," IEEE Transactions on Sustainable Energy, vol. 6, no. 2, pp. 635-643, Apr. 2015, ISSN: 1949-3029. DOI: 10.1109/TSTE.2015. 2402834

[21] M. Mehrasa, E. Pouresmaeil, H. Mehrjerdi, B. N. J, and J. P. Catalão, "Control technique for enhancing the stable operation of distributed generation units within a microgrid," Energy Conversion and Management, vol. 97, pp. 362373, 2015, ISSN: 0196-8904. DOI: http://dx.doi.org/10.1016/j.enconman.2015.03.078.

[22] M. Rahman, M. Hossain, and J. Lu, "Utilization of parked ev-ess for power management in a grid-tied hybrid ac/dc microgrid," in 2015 Australasian Universities Power Engineering Conference (AUPEC), Sep. 2015, pp. 1-6. DOI: 10. 1109/AUPEC. 2015.7324812.

[23] F. Rafi, M. Hossain, and J. Lu, "Design of a single stage transformerless vsi in a smart microgrid for pv-statcom/ess operations," in 2014 Australasian Universities Power Engineering Conference (AUPEC), Sep. 2014, pp. 1-6. DOI: 10. 1109/AUPEC. 2014.6966557.

[24] D. Leskarac, C. Bennett, M. Moghimi, S. Stegen, and J. Lu, "Testing facility for research and development of smartmicrogrid technologies," in 2015 IEEE PES Asia-Pacific Power and Energy Engineering Conference (APPEEC), Nov. 2015, pp. 1-5. DOI: 10.1109/APPEEC. 2015.7381059.

[25] M. Moghimi, C. Bennett, D. Leskarac, S. Stegen, and J. Lu, "Communication architecture and data acquisition for experimental microgrid installations," in 2015 IEEE PES Asia-Pacific Power and Energy Engineering Conference (APPEEC), Nov. 2015, pp. 1-5. DOI: 10.1109/APPEEC.2015.7380917.

[26] T. Alnejaili, S. Drid, D. Mehdi, L. Chrifi-Alaoui, R. Belarbi, and A. Hamdouni, "Dynamic control and advanced load management of a stand-alone hybrid renewable power system for remote housing," Energy Conversion and Management, vol. 105, pp. 377-392, 2015, ISSN: 0196-8904. DOI: http://dx.doi.org/10.1016/j.enconman.2015.07.080.

[27] S. Koohi-Kamali, N. Rahim, and H. Mokhlis, "Smart power management algorithm in microgrid consisting of photovoltaic, diesel, and battery storage plants considering variations in sunlight, temperature, and load," Energy Conversion and Management, vol. 84, pp. 562-582, 2014, ISSN: 0196-8904. DOI: http://dx.doi.org/10.1016/j.enconman.2014.04. 072. 
[28] M. A. Mahmud, H. R. Pota, and M. J. Hossain, "Dynamic stability of three-phase grid-connected photovoltaic system using zero dynamic design approach," IEEE Journal of Photovoltaics, vol. 2, no. 4, pp. 564-571, Oct. 2012, ISSN: 21563381. DOI: 10.1109/JPHOTOV.2012.2195551.

[29] R. Kroeze and P. Krein, "Electrical battery model for use in dynamic electric vehicle simulations," in IEEE Power Electronics Specialists Conference PESC 2008, Jun. 2008, pp. 1336-1342. DOI: 10.1109/PESC.2008.4592119.

[30] R. Minciardi and M. Robba, "A dynamic model for electrical vehicles interacting with microgrids and renewables," in 2013 European Modelling Symposium (EMS), Nov. 2013, pp. 420-425. DOI: 10.1109/EMS.2013.71.

[31] O. Tremblay and L. A. Dessaint, "Experimental validation of a battery dynamic model for ev applications," J. World Electric Vehicle, vol. 3, 2009.

[32] AmirnaserYazdani and R. Iravani, Voltage-sourced converters in power systems: modeling, control, and applications. John Wiley \& Sons, 2010.

[33] J. Guerrero, J. Vasquez, J. Matas, L. de Vicuna, and M. Castilla, "Hierarchical control of droop-controlled ac and dc microgrids - a general approach toward standardization," IEEE Transactions on Industrial Electronics, vol. 58, no. 1, pp. 158-172, Jan. 2011, ISSN: 0278-0046. DOI: 10.1109/TIE.2010.2066534.

[34] J. M. Guerrero, J. C. Vasquez, J. Matas, M. Castilla, and L. G. de Vicuna, "Control strategy for flexible microgrid based on parallel line-interactive ups systems," IEEE Transactions on Industrial Electronics, vol. 56, no. 3, pp. 726-736, Mar. 2009, ISSN: 0278-0046. DOI: 10.1109/TIE.2008.2009274.

[35] T. Morstyn, B. Hredzak, and V. Agelidis, "Distributed cooperative control of microgrid storage," IEEE Transactions on Power Systems, vol. 30, no. 5, pp. 2780-2789, Sep. 2015, ISSN: 0885-8950. DOI: 10.1109/TPWRS.2014.2363874.

[36] Parliament of the Commonwealth of Australia and E. Act, "Electricity Regulation 2006," Tech. Rep. 4, 2011.

[37] T. A. E. M. C. Reliability Panel, "Application of Frequency Operating Standards During Periods of Supply Scarcity," Tech. Rep. April, 2009.

[38] T. A. P. Association, "Pv integration on australian distribution," no. September, 2013.

[39] N. Pogaku, M. Prodanovic, and T. C. Green, "Modeling, analysis and testing of autonomous operation of an inverterbased microgrid," IEEE Transactions on Power Electronics, vol. 22, no. 2, pp. 613-625, 2007, ISSN: 08858993. DOI: 10.1109/TPEL. 2006.890003.

[40] P. H. Divshali, A. Alimardani, S. H. Hosseinian, and M. Abedi, "Decentralized cooperative control strategy of microsources for stabilizing autonomous vsc-based microgrids," IEEE Transactions on Power Systems, vol. 27, no. 4, pp. 1949-1959, Nov. 2012, ISSN: 0885-8950. DOI: 10.1109/TPWRS.2012.2188914.

[41] R. J. Hamidi, H. Livani, S. Hosseinian, and G. Gharehpetian, "Distributed cooperative control system for smart microgrids," Electric Power Systems Research, vol. 130, pp. 241-250, 2016, ISSN: 0378-7796. DOI: http://dx.doi.org/10. 1016/j.epsr.2015.09.012.

[42] A. A. A. Radwan and Y. A. R. I. Mohamed, "Networked control and power management of ac/dc hybrid microgrids," IEEE Systems Journal, vol. PP, no. 99, pp. 1-12, 2014, ISSN: 1932-8184. DOI: 10.1109/JSYST.2014.2337353.

[43] B. Singh, P. Jayaprakash, T. R. Somayajulu, and D. P. Kothari, "Reduced rating vsc with a zig-zag transformer for current compensation in a three-phase four-wire distribution system," IEEE Transactions on Power Delivery, vol. 24, no. 1, pp. 249-259, Jan. 2009, ISSN: 0885-8977. DOI: 10.1109/TPWRD.2008.2005398.

[44] J. P. Torreglosa, P. García-Triviño, L. M. Fernàndez-Ramirez, and F. Jurado, "Decentralized energy management strategy based on predictive controllers for a medium voltage direct current photovoltaic electric vehicle charging station," Energy Conversion and Management, vol. 108, pp. 1-13, 2016, ISSN: 0196-8904. DOI: http://dx.doi.org/10.1016/j.enconman. 2015.10.074.

[45] N. Bottrell, M. Prodanovic, and T. C. Green, "Dynamic stability of a microgrid with an active load," IEEE Transactions on Power Electronics, vol. 28, no. 11, pp. 5107-5119, Nov. 2013, ISSN: 0885-8993. DOI: 10.1109/TPEL.2013.2241455. 